\title{
ANALYTIC WAVE FRONT SETS FOR SOLUTIONS OF LINEAR DIFFERENTIAL EQUATIONS OF PRINCIPAL TYPE
}

BY

\author{
KARL GUSTAV ANDERSSON
}

ABSTRACT. The propagation of analyticity for solutions $u$ of $P(x, D) u=f$ is studied, in terms of wave front sets, for a large class of differential operators $P=P(x, D)$ of principal type. In view of a theorem by L. Hörmander [9], the results obtained imply rather precise results about the surjectivity of the mapping $P: C^{\infty}(\boldsymbol{\Omega}) \rightarrow C^{\infty}(\boldsymbol{\Omega})$.

Introduction. Let $P=P(x, D)$ be a linear differential operator with $C^{\infty}$-coefficients in an open set $\Omega \subset R^{n}$. When $P$ is elliptic, then the classical regularity theorem for elliptic equations says that the distribution $u$ is infinitely differentiable whenever $P u$ is and, if the coefficients of $P$ are analytic, then $u$ is analytic where $P u$ is analytic. The corresponding question, for more general operators, of describing the set of singularities of $u$ when that of $P u$ is given has been much studied lately (see [10] and the references there). The introduction of the concept of wave front sets (see [7] and [14]) has added precision to the statements and has also simplified many proofs. For operators with real principal part $P_{m}(x, D)$, such that the Hamilton field

$$
H_{P_{m}}=\sum_{1 \leq j \leq n}\left[\left(\partial P_{m}(x, \xi) / \partial \xi_{j}\right) \partial / \partial x_{i}-\left(\partial P_{m}(x, \xi) / \partial x_{j}\right) \partial / \partial \xi_{j}\right]
$$

is nondegenerate when $\xi \in \dot{R}^{n}=R^{n} \backslash\{0\}$, and for some operators with complex coefficients, very complete results for the $C^{\infty}$-case are obtained by Duistermaat and Hörmander in [5] (see also [17]). Corresponding results, concerning analyticity, are proved in [1] (operators with constant coefficients), [9] and [11], under the assumption that $P_{m}$ is real and $d_{\xi} P_{m}(x, \xi)=\left(\partial P_{m}(x, \xi) / \partial \xi_{1}, \cdots\right.$, $\left.\partial P_{m}(x, \xi) / \partial \xi_{n}\right) \neq 0$ in $\Omega \times \dot{R}^{n}$. The purpose of this paper is to extend the results in the analytic case to certain operators with complex coefficients. In doing so, we shall also give new proofs for operators with real principal part. The argument is modeled on the very elegant proof, in the $C^{\infty}$-case, which Hörmander gave in his Congress lecture [8] for operators with real principal part. The main results

Received by the editors February 1, 1972.

AMS (MOS) subject classifications (1970). Primary 35A05, 35A20.

Key words and phrases. Analytic wave front sets, pseudo-differential operators. 
are stated in $\$ 1$, and in $\$ 2$ we define certain sequences of pseudo-differential operators used to localize the problem in $T^{*}(\Omega) . \$ 3$ contains a proof of the noncharacteristic regularity theorem and two lemmas about the existence of sequences of pseudo-differential operators suitable for the localization. To obtain the local regularity theorem, we use a variant of a classical inequality of Calderón [4], proved in $\$ 4$, and in $\$ 5$ the proofs of the theorems stated in $\$ 1$ are completed. The results of this paper have been announced in [2].

1. Statement of the main results. Let $K$ be a compact subset of the open set $\Omega \subset R^{n}$. Then it is well known (see e.g. [1] and the references there) that there are functions $\phi_{N} \in C_{0}^{\infty}(\Omega)$ which are equal to 1 on $K$ and, for some constant $C$, satisfy

$$
\left|D^{a} \phi_{N}(x)\right| \leq C(C N)|a|, \text { when }|a| \leq N .
$$

It is easy to see (compare [9]) that a distribution $u$ is analytic in a neighborhood of $x_{0} \in \Omega$ if and only if there is such a sequence $\left(\phi_{N}\right)$ with all $\phi_{N}=1$ on a neighborhood of $x_{0}$ and

$$
\left.\mid \widehat{\left(\phi_{N} u\right.}\right)(\xi) \mid \leq C^{N}(1+|\xi| / N)^{-N} \text {. }
$$

This motivates the following definition of the analytic wave front set, WF ${ }_{a}(u)$, of a distribution $u$.

Definition 1.1. Let $u \in \mathscr{D}^{\prime}(\Omega)$ and $\left(x_{0}, \xi_{0}\right) \in \Omega \times \dot{R}^{n}$. We say that $\left(x_{0}, \xi_{0}\right) \notin$ WF $a_{a}(u)$ if and only if there is a neighborhood $U$ of $x_{0}$ and a sequence $u_{N} \in \mathcal{E}^{\prime}(\Omega)$ such that $u_{N}=u$ in $U$ and

$$
\left|\hat{u}_{N}(\xi)\right| \leq C^{N}(1+|\xi| / N)^{-N}
$$

where $\xi$ belongs to some fixed conic neighborhood of $\xi_{0}$.

Remark 1.1. Definition 2.1 is readily seen to be equivalent to the definition of WF $a_{a}(u)$ given in [9]. There is also available the set supp sp $u$ (see [16]) whose definition, for a hyperfunction $u$, was indicated by M. Sato in [14] (see also [15]). Probably WF $\mathbb{W}_{a}(u)=\operatorname{supp} \mathrm{sp} u$, when $u$ is a distribution, but so far this has not been proved.

WF ${ }_{a}(u)$ is thus a subset of $\Omega \times \dot{R}^{n}$ or, if one wants to emphasize the behavior under coordinate transformations, $\dot{T}^{*}(\Omega)$, where the dot indicates that the zerosection is removed. More precisely, it is proved in [9] that, if $y=\kappa(x)$ is an analytic change of coordinates in $\Omega$, and $\beta$ denotes the map on $\Omega \times \dot{R}^{n}$ defined by $\beta(x, \xi)=\left(\kappa(x),\left({ }^{t} \kappa^{\prime}(x)\right)^{-1}(\xi)\right)$, then $\mathbb{W F}_{a}\left(u \circ \kappa^{-1}\right)=\beta\left(\mathbb{W F}_{a}(u)\right)$. This also follows directly from Theorem 2.3 and the alternative characterization of $\mathbb{W F}_{a}(u)$ which is given in $\$ 3$. Denote by $\pi$ the projection $T^{*}(\Omega) \rightarrow \Omega$ and by a.s. $u$ the complement of the largest open subset of $\Omega$ on which $u$ is analytic. Then 


$$
\pi\left(W_{a}(u)\right)=\text { a.s. } u
$$

(for a proof, see [9]). Now let $P=P(x, D)$ be linear differential operator in $\Omega$ with analytic coefficients and denote by $Z\left(P_{m}\right)^{a}$ the set $\left\{(x, \xi) \in \Omega \times \dot{R}^{n}\right.$; $\left.P_{m}(x, \xi)=0\right\}$. Then we have the following generalization of the elliptic regularity theorem.

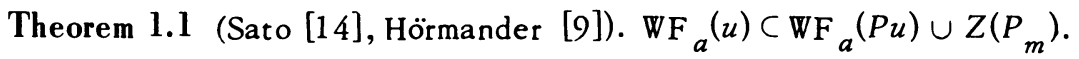

Note that if $P$ is elliptic and $P u$ analytic, then the right-hand side is empty, so (1.4) implies that $u$ is analytic. In $\$ 3$ we shall prove a result, Lemma 3.1, which is slightly stronger than Theorem 1.1 .

The more precise results about propagation of singularities inside $Z\left(P_{n}\right)$ will depend on the properties of the bicharacteristics of $P$. If $P_{m}$ is real and $H_{P_{m}}$ is nondegenerate, then $Z\left(P_{m}\right)$ is a manifold and $H_{P_{m}}$ is tangent to $Z\left(P_{m}\right)$. The integral curves in $Z\left(P_{m}\right)$ corresponding to $H_{P_{m}}$ are called the bicharacteristic strips of $P$ and the ir projections on $\Omega$ are called bicharacteristic curves. If, in addition, $d_{\xi} P_{m} \neq 0$ on a bicharacteristic strip, then the corresponding bicharacteristic curve is regular. We denote by $N_{1}\left(P_{m}\right)$ the set $\left\{(x, \xi) \in Z\left(P_{m}\right)\right.$; $\left.d_{\xi} P_{m}(x, \xi) \neq 0\right\}$.

Theorem 1.2. If $P=P(x, D)$ bas analytic coefficients and real principal part $P_{m}(x, D)$, then

$$
\text { WF } a_{a}(u) \text { is invariant under } H_{P_{m}} \text { in } N_{1}\left(P_{m}\right) \backslash \mathbb{W} \mathrm{F}_{a}(P u) .
$$

When $P$ is principal type, i.e., $N_{1}\left(P_{m}\right)=Z\left(P_{m}\right)$, and has real principal part, then Theorem 1.1 and Theorem 1.2 together with (1.4) immediately imply the following result.

Suppose that the distribution $u$ is analytic in $\Omega_{0} \subset \Omega$. Then $u$ is analytic in a neighborhood of any point $x_{0}$ such that, for every bicharacteristic curve $l$ through $x_{0}$, the component of $(\Omega \cap l) \backslash($ a.s. $P u)$ which contains $x_{0}$ also contains some point in $\Omega_{0}$.

This result was proved for operators with constant coefficients in [1]. For general operators with analytic coefficients the more precise Theorem 1.2 has been proved by Hörmander [9] and, for hyperfunctions and Sato's wave front sets, by Kawai-Kashiwara (see [11]). We shall give another proof of Theorem 1.2.

When $P_{m}$ has complex coefficients, then $Z\left(P_{m}\right)$ is a manifold of codimension 2, provided that the vector fields $H_{\operatorname{Re}} P_{m}$ and $H_{\operatorname{Im}} P_{m}$ are linearly independent. If, in addition, the Poisson bracket $\left\{\operatorname{Re} P_{m}^{m}, \operatorname{Im} P_{m}\right\}=H_{\operatorname{Re} P_{m}}\left(\operatorname{Im} P_{m}\right)$ vanishes in $Z\left(P_{m}\right)$ then the vector fields $H_{\mathrm{Re}} P_{m}$ and $H_{\mathrm{Im}} P_{m}$ are tangent to $Z\left(P_{m}\right)$ and, because of the Frobenius integrability theorem, they define a 2-dimensional foliation 
of $Z\left(P_{m}\right)$. This foliation is called the bicharacteristic foliation and its leaves are called the bicharacteristic strips of $P$. If $d_{\xi} \operatorname{Re} \grave{P}_{m}$ and $d_{\xi} \operatorname{Im} P_{m}$ are linearly independent on a bicharacteristic strip, then its projection on $\Omega$ is a regular 2 -dimensional manifold. We put

$$
N_{2}\left(P_{m}\right)=N_{2}^{\prime}\left(P_{m}\right) \cap N_{2}^{\prime \prime}\left(P_{m}\right)
$$

where

$$
N_{2}^{\prime}\left(P_{m}\right)=\left\{(x, \xi) \in Z\left(P_{m}\right) ; d_{\xi} \operatorname{Re} P_{m}(x, \xi) \text { and } d_{\xi} \operatorname{Im} P_{m}(x, \xi)\right. \text { are }
$$

linearly independent $\}$.

$$
\begin{array}{r}
N_{2}^{\prime \prime}\left(P_{m}\right)=\left\{\left(x_{0}, \xi_{0}\right) \in Z\left(P_{m}\right) ;\left\{\operatorname{Re} P_{m}, \operatorname{Im} P_{m}\right\}(x, \xi)=0\right. \\
\text { in a neighborhood, } \\
\text { in } \left.Z\left(P_{m}\right), \text { of }\left(x_{0}, \xi_{0}\right)\right\} .
\end{array}
$$

Theorem 1.3. If $P=P(x, D)$ bas analytic coefficients, then $\mathbb{W F}_{a}(u)$ is invariant under the bicharacteristic foliation in $N_{2}\left(P_{m}\right) \backslash \mathbb{W} F_{a}(P u)$.

Remark 1.2. T. Kawai has announced (private communication) that, by extending the theory of Fourier integral operators to the analytic category, he and M. Kashiwara have proved Theorem 1.3 for hyperfunctions. The proof we shall give has quite a different character, since only pseudo-differential operators are used.

A third case which we shall consider is when $P=P(D)$ has constant coefficients. Then

$$
H_{P_{m}}=\sum_{1 \leq j \leq n}\left(\partial P_{m}(\xi) / \partial \xi_{j}\right) \partial / \partial x_{j}
$$

is a differential operator in $\Omega$ with constant coefficients depending on the parameter $\xi$. If

$$
\left(x_{0}, \xi_{0}\right) \in N_{1}\left(P_{m}\right)=\Omega \times\left\{\xi \in \dot{R}^{n} ; P_{m}(\xi)=0 \text { and } d_{\xi} P_{m}(\xi) \neq 0\right\}
$$

then this operator is nondegenerate and the bicharacteristic strip through $\left(x_{0}, \xi_{0}\right)$ is defined to be $\left\{\left(x_{0}+d_{\xi}\left(z P_{m}\right)\left(\xi_{0}\right), \xi_{0}\right) ; z \in \mathbf{C}\right\}$. These bicharacteristic strips define a foliation, the bicharacteristic foliation of $N_{1}\left(P_{m}\right)$. Note that the dimension of the leaves may vary between 1 and 2 depending on whether $d_{\xi} \operatorname{Re} P_{m}$ and $d_{\xi} \operatorname{Im} P_{m}$ are linearly dependent or not.

Theorem 1.4. If $P=P(D)$ bas constant coefficients, then $\mathrm{WF}_{a}(u)$ is invariant under the bicharacteristic foliation in $N_{1}\left(P_{m}\right) \backslash \mathbb{W F} a(P u)$.

Theorems 1.1-1.3 supplement the results of Duistermaat-Hörmander [5] for operators with analytic coefficients, provided that the projections of the bicharacteristic strips are regular. Then it follows directly from Theorems 1.1- 
1.3 that the conditions imposed on $P$ and $\Omega$ in [5] to prove that $P$ maps $\mathscr{D}^{\prime}(\Omega) / C^{\infty}(\Omega)$ onto itself actually give that $P C^{\infty}(\Omega)=C^{\infty}(\Omega)$ and thus $P \mathscr{D}^{\prime}(\Omega)=\mathscr{D}^{\prime}(\Omega)$. However, for operators with analytic coefficients, $P C^{\infty}(\Omega)=C^{\infty}(\Omega)$ under much weaker assumptions. In fact, Hörmander [9] observed that results about propagation of analyticity for wave front sets can be used to derive very precise uniqueness theorems. He proved the following general result.

Theorem 1.5 (Hörmander [9]). Suppose that $\Omega_{0}$ is an open subset of $\Omega$ with $C^{1}$ boundary $\partial \Omega_{0^{\circ}}$. Denote by $N_{0}$ the normal of $\partial \Omega_{0}$ at $x_{0} \in \Omega$ and let $F$ be a conic neigbborbood of $\left(x_{0}, \pm N_{0}\right)$. Then there is a neigbborbood $\Omega^{\prime}$ of $x_{0}$ such that any $u \in \mathscr{D}^{\prime}(\Omega)$, with $\mathrm{WF}_{a}(u) \cap F=\varnothing$, which vanishes in $\Omega_{0}$ must also vanis $h^{\prime}$ in $\Omega^{\prime}$.

To be brief, we just state what this theorem together with Theorem 1.4 implies for operators with constant coefficients.

Theorem 1.6. Let $\Omega \subset R^{n}$ be an open set with $C^{1}$ boundary $\partial \Omega$ such that all points $x_{0} \in \partial \Omega$, characteristic with respect to $P(D)$, are simply characteristic, i.e. $d_{\xi} P_{m}\left(N_{0}\right) \neq 0$ for the normal $N_{0}$ of $\partial \Omega$ at $x_{0}$. Denote by $B_{x_{0}}$ the projection of the bicharacteristic strip through $\left(x_{0}, N_{0}\right)$ and by $\hat{K}$ the convex bull of a compact set $K$. If, for any characteristic $x_{0} \in \partial \Omega$ and any compact set $K \subset \Omega$, the component of $B_{x_{0}} \cap \mathrm{C}_{K}$ containing $x_{0}$ also contains some point in $\mathrm{C}(\bar{\Omega} \cap \hat{K})$ then $P(D) C^{\infty}(\Omega)=C^{\infty}(\Omega)$ and, what is equivalent, $P(D) \mathscr{D}_{F}^{\prime}(\Omega)=\mathscr{D}_{F}^{\prime}(\Omega)$.

This result improves Theorem 1.3 .7 of [10].

2. A space of sequences of pseudo-differential operators. Functions $\phi_{N}$ satisfying (1.1) are suitable for the localization of problems concerning analytic functions in $\Omega$. We shall now define certain sequences of symbols which will fill the same purpose in the cotangent space $T^{*}(\Omega)$.

Definition 2.1. A sequence $\left(a_{N}(x, \xi)\right)$ is said to belong to $\tau^{r}(\Omega)$ if, for every compact set $K \subset \Omega$, there are positive constants $\delta$ and $C$ such that, if $N \geq \delta^{-2}$, $a_{N} \in C^{[\delta N]}\left(K \times R^{n}\right)$ and

$$
\sup _{x \in K}\left|D_{x}^{a} D_{\xi}^{\beta} a_{N}(x, \xi)\right| \leq C^{N}|\alpha|(1+|\xi| / N)^{r-|B|,} \text { when }|\alpha+\beta| \leq \delta N .
$$

Remark 2:1. The reason for the presence of the constant $\delta$ in Definition 2.1 is purely technical. For example, the right-hand side of (2.6) will in general admit fewer derivatives than $a_{N}$ and $b_{N}$. However, by putting $\left.a_{N}^{\prime}=a_{[N} \cdot \delta-2\right]_{+1}$, we can always obtain a sequence for which $\delta=1$.

Example 2.1. Let $P(x, D)$ be a linear differential operator of order $r$ with analytic coefficients in $\Omega$ and put $a_{N}(x, \xi)=P(x, \xi)$. Then $\left(a_{N}\right) \in \tau^{r}(\Omega)$. 
Example 2.2. Suppose that the functions $\phi_{N}(x)$ satisfy $(1.1)$ in $\Omega$ and that $\psi_{N}(\xi)$ are homogeneous functions of degree $r$, satisfying (1.1) when $|\xi|=1$. If $\chi_{N}$ vanishes when $|\xi| \leq 1$, equals 1 when $|\xi| \geq 2$ and satisfies (1.1) then $\left(\phi_{N}(x) \psi_{N}(\xi) \chi_{N}(\xi / N)\right) \in r^{r}(\Omega)$. For convenience, we shall introduce a special notation for sequences of this type. Let $F$ and $F^{\prime}$ be two open cones in $\Omega \times \dot{R}^{n}$, i.e., open subsets of $\Omega \times \dot{R}^{n}$ which are invariant under multiplication of the $\xi$ coordinate with a positive number. Suppose that $\dot{\bar{F}} \subset F^{\prime}$ and denote by $\tau^{0}\left(F, F^{\prime}\right)$ the set of sequences $\left(a_{N}\right) \in \tau^{0}(\Omega)$ such that, for some $C>0, a_{N}(x, \xi)$ vanishes in $\left(\mathbf{C} F^{\prime}\right) \cup\{(x, \xi) ;|\xi| \leq C N\}$ and $a_{N}(x, \xi)=1$ in $F \cap\{(x, \xi) ;|\xi| \geq 2 C N\}$.

Example 2.3. Suppose that $F^{\prime} \subset \Omega \times \dot{R}^{n}$ is an open cone such that $\pi\left(F^{\prime}\right)=$ $\left\{x ;(x, \xi) \in F^{\prime}\right\} \subset \subset \Omega$, i.e., $\overline{\pi\left(F^{\prime}\right)}$ is a compact subset of $\Omega$, and let $p$ be an analytic symbol of order $r$ in $F^{\prime}$, in the sense of Boutet de Monvel and Krée [3]. This means that $p$ is a formal sum $p=\Sigma_{-\infty<k \leq r} p^{k}$ with the property that for every cone $F$, with $\bar{F} \subset F^{\prime}$, there is a constant $C>0$ such that

$$
\left|D_{x}^{\alpha} D_{\xi}^{\beta} p^{k}(x, \xi)\right| \leq C^{\left|a_{+} \beta\right|+|k|+1}|k| !|\alpha| !|\beta||\xi|^{k-|\beta|}, \quad \text { when }(x, \xi) \in F .
$$

Moreover, it is assumed that $p^{k}(x, \xi)$ is homogeneous of degree $k$ with respect to $\xi$. If $\left(\mu_{N}(x, \xi)\right) \in \tau^{0}\left(F, F^{\prime}\right)$ for some open cone $F$, with $\overline{\bar{F}} \subset F^{\prime}$, then $\left(p_{N}(x, \xi)\right)=\left(\Sigma_{-N \leq k \leq r} p^{k}(x, \xi) \mu_{N}(x, \xi)\right) \in \tau^{r}(\Omega)$. Note that it follows from (2.2) that $p^{k}(x, \xi)$ is an analytic function in $F^{\prime}$. In order to obtain nontrivial symbols with support in a compactly generated cone, we therefore have to consider the sequences in $\tau^{r}(\Omega)$.

If $\left(a_{N}\right) \in \tau^{r}(\Omega), \Omega^{\prime} \subset \subset \Omega$ and $\delta=\delta\left(\Omega^{\prime}\right)$ is small enough, then $a_{N}$ defines a mapping $C_{0}^{\infty}\left(\Omega^{\prime}\right) \rightarrow C^{[\delta N]}\left(\Omega^{\prime}\right)$ by means of the formula

$$
a_{N}(x, D) u(x)=(2 \pi)^{-n} \int e^{i(x, \xi)} a_{N}(x, \xi) u(\xi) d \xi, \quad u \in C_{0}^{\infty}\left(\Omega^{\prime}\right)
$$

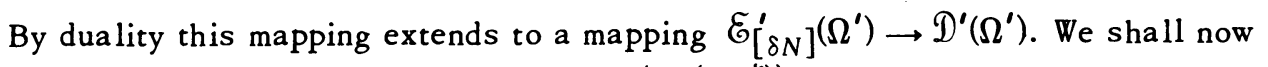
give conditions on the symbol sequence $\left(a_{N}(x, \xi)\right)$ which will ensure that the sequence of operators defined by (2.3) is regularizing in the following sense. For any $u \in \mathcal{E}^{\prime}(\Omega)$ and any $\Omega^{\prime} \subset \subset \Omega$ there are constants $\delta$ and $C$ such that $a_{N}(x, D) u(x) \in C^{[\delta N]}\left(\Omega^{\prime}\right)$, when $N \geq \delta^{-2}$, and

$$
\sup _{x \in \Omega^{\prime}}\left|D_{x}^{a} a_{N}(x, D) u(x)\right| \leq C^{N} N^{|a|}, \quad \text { when }|a| \leq \delta N .
$$

Definition 2.2. We say that the sequence $\left(a_{N}(x, \xi)\right)$ is in $\tau_{0}(\Omega)$ if, for every compact set $K \subset \Omega$, there are positive constants $\delta$ and $C$ such that, if $N \geq \delta^{-2}$, $a_{N} \in C^{[\delta N]}\left(K \times R^{n}\right)$ and

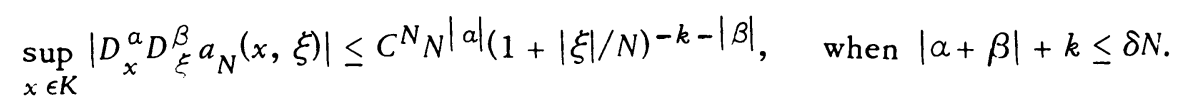


If $\left(a_{N}\right),\left(b_{N}\right) \in \tau^{r}(\Omega)$ and $\left(a_{N}-b_{N}\right) \in \tau_{0}(\Omega)$, we write $\left(a_{N}\right) \sim\left(b_{N}\right)$.

Remark 2.2. Obviously $\tau_{0}(\Omega) \subset \bigcap_{r} \tau^{r}(\Omega)$. However, much more information is provided by (2.3). In particular it is clear that $\tau_{0}(\Omega)$ is a proper subset of $\bigcap_{r} \tau^{r}(\Omega)$.

Example 2.4. Let $\left(\chi_{N}(\xi)\right)$ be a sequence of functions satisfying (1.1) and vanishing when $|\xi|>C>0$. Then $\left(\chi_{N}(\xi / N)\right) \in \tau_{0}\left(R^{n}\right)$.

We shall now give a calculus for the sequences of pseudo-differential operators associated with the spaces $\tau^{r}(\Omega)$. The treatment will contain nothing essentially new beyond "classical" expositions of the calculus of pseudo-differential operators. We shall, therefore, be brief at some points and refer to [6], [7], and [13] for the calculus of pseudo-differential operators.

Theorem 2.1. Let $\left(a_{N}\right) \in \tau^{r}(\Omega)$ and $\left(b_{N}\right) \in \tau^{s}(\Omega)$. Suppose that $b_{N}(x, \xi)$ vanishes when $x$ is outside a fixed compact set $K \subset \Omega$. Then there is a sequence $\left(\dot{a}_{N} \circ b_{N}\right) \in \tau^{r+s}(\Omega)$ such that when $u \in C_{0}^{\infty}(\Omega)$ and $N$ is large, then $\left(a_{N} \circ b_{N}\right)(x, D) u=a_{N}(x, D)\left(b_{N}(x, D) u\right)$. If $\epsilon$ is small enough, we have

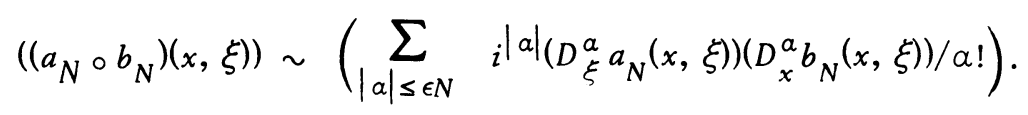

Proof. Put

$$
v_{N}(x)=b_{N}(x, D) u(x)=(2 \pi)^{-n} \int e^{i(x, \xi)} b_{N}(x, \xi) \hat{u}(\xi) d \xi
$$

If $N$ is large, $a_{N}(x, D) v_{N}(x)$ is well defined and we may operate with $a_{N}(x, D)$ under the sign of integration. Therefore, $\left(a_{N} \circ b_{N}\right)(x, \xi)=a_{N}(x, D+\xi) b_{N}(x, \xi)$ and we only have to prove that

$$
\left(r_{N}\right)=\left(a_{N} \circ b_{N}-\sum_{|x| \leq \epsilon N} i|a|\left(D_{\xi}^{a} a_{N}\right)\left(D_{x}^{a} b_{N}\right) / \alpha !\right)
$$

belongs to $\tau_{0}(\Omega)$. If $\hat{b}_{N}$ denotes the Fourier transform of $b_{N}$ with respect to $x$, then Taylor's formula gives that

where

$$
r_{N}(x, \xi)=(2 \pi)^{-n} \int r_{N}(x, \xi, \eta) d \eta
$$

$$
\begin{aligned}
{ }_{N}(x, \xi, \eta)=\sum_{|\gamma|=[\epsilon N]+1}{ }_{i}|\gamma|_{e^{i\langle x, \eta\rangle}\left(\eta^{\gamma} \hat{b}_{N}(\eta, \xi) / \gamma !\right)} & \\
& \cdot\left(\int_{0}^{1} D_{\xi}^{\gamma} a_{N}(x, \xi+t \eta)(1-t)|\gamma|-1 d t\right) .
\end{aligned}
$$

When $x$ belongs to a compact set $K$ and $2|\eta| \leq|\xi|$, then (2.1) implies

$$
|\xi|^{k}\left|D_{x}^{a^{\prime}} D_{\xi}^{\beta^{\prime}+\gamma} a_{N}(x, \xi+t \eta)\right| \leq C_{1}^{N}{ }_{N}|a|+k(1+|\xi| / N)^{r+k-|\gamma|-\left|\beta^{\prime}\right|}
$$

and 


$$
\left|\eta^{a^{\prime \prime}+\gamma} D_{\xi}^{\beta^{\prime \prime}} \hat{b}_{N}(\eta, \xi) / \gamma !\right| \leq C_{2}^{N} N^{\left|a^{\prime \prime}\right|+|\gamma|} /\left.(1+|\xi| / N)\right|^{\prime \prime \prime} \mid(1+|\eta|)^{n+1} \gamma !
$$

Thus if $|\alpha|=\left|\alpha^{\prime}\right|+\left|\alpha^{\prime \prime}\right|,|\beta|=\left|\beta^{\prime}\right|+\left|\beta^{\prime \prime}\right|$ and $r+k \leq|\gamma|=[\epsilon N]$, we have

$$
|\xi|^{k}\left|D_{x}^{\alpha} D_{\xi}^{\beta} r_{N}(x, \xi, \eta)\right| \leq\left(C_{3}^{N} N^{|\alpha|+k} /(1+|\xi| / N)|\beta|(1+|\eta|)^{n+1}\left({ }_{N}|\gamma| / \gamma !\right)\right.
$$

when $2|\eta| \leq|\xi|$. On the other hand (2.1) implies the following inequalities when $x \in K$ and $|\xi| \leq 2|\eta|$ :

$$
\begin{aligned}
&\left|D_{x}^{a^{\prime}} D_{\xi}^{\beta^{\prime}+\gamma} a_{N}(x, \xi+t \eta)\right| \leq C_{4}^{N} N_{N}\left|a^{\prime}\right| \\
&|\xi|^{j}\left|\eta^{\alpha^{\prime \prime}+\gamma} D_{\xi}^{\beta^{\prime \prime} \hat{b}_{N}}(\eta, \xi)\right| \leq C_{s}^{N}{ }_{s}\left|a^{\prime \prime}\right|+j+|\gamma| /(1+|\xi| / N)\left|\beta^{\prime \prime}\right| \\
&(1+|\eta|)^{n+1} \gamma ! .
\end{aligned}
$$

By choosing $j=k+\left|\beta^{\prime}\right|$ and $j=k$ we see that (2.9) is valid also when $|\xi| \leq$ $2|\eta|$, perhaps with another constant. This proves Theorem 2.1 , if we also note that $N^{|\gamma|} / \gamma ! \leq N^{N} / N ! \leq C^{N}$.

Suppose now that $\left(a_{N}\right) \in \tau^{r}(\Omega)$ and let $a_{N}(x, D)$ be the mapping defined by (2.3). The distribution kernel of this mapping is defined by

$$
K_{N}(w)=(2 \pi)^{-n} \iint e^{i(x, \xi)} a_{N}(x, \xi) \hat{w}(x, \xi) d x d \xi, \quad w \in C_{0}^{\infty}(\Omega \times \Omega),
$$

where $\hat{w}$ denotes Fourier transform with respect to the second variable. For any compact set $M$ in $\Omega \times \Omega$, which does not intersect the diagonal, there are positive constants $\delta$ and $C$ such that $K_{N} \in C^{[\delta N]}(M)$, when $N \geq \delta^{-2}$, and

$$
\sup _{x, y \in M}\left|D_{x}^{a} D_{y}^{\beta} K_{N}(x, y)\right| \leq C^{N}|\alpha|+|\beta|, \quad \text { when }|\alpha|+|\beta| \leq \delta N .
$$

For sequences $\left(a_{N}\right) \in \tau_{0}(\Omega)$ the kernel $K_{N}$ belongs to $C^{[\delta N]}(M)$ and satisfies (2.13) for any compact set $M \subset \Omega \times \Omega$, if $N \geq \delta^{-2}=\delta(M)^{-2}$. Conversely, suppose that $\left(K_{N}(x, y)\right)$ is a sequence such that, for every compact set $M \subset \Omega \times \Omega$, there are positive constants $\delta$ and $C$ such that $K_{N} \in C^{[\delta N]}(M)$ and satisfies (2.13) when $N \geq \delta^{-2}$. If in addition the functions $K_{N}(x, y)$ vanish when $y$ is outside a fixed compact set, then the sequence $\left(a_{N}(x, \xi)\right)=\left(e^{-i(x, \xi)} \int K_{N}(x, y) e^{i(y, \xi)} d y\right)$ belongs to $\tau_{0}(\Omega)$ and

$$
a_{N}(x, D) u(x)=\int K_{N}(x, y) u(y) d y .
$$

However, even if there is no compact set $L \subset \Omega$ such that all $K_{N}(x, y)$ vanish when $y \notin L$, the mapping $C_{0}^{\infty}\left(\Omega^{\prime}\right) \rightarrow D^{\prime}\left(\Omega^{\prime}\right)$ given by

$$
u \mapsto \int K_{N}(\cdot, y) u(y) d y, \quad u \in C_{0}^{\infty}\left(\Omega^{\prime}\right),
$$

is well defined for any $\Omega^{\prime} \subset \subset \Omega$ if $N$ is large enough. We denote by $T_{0}(\Omega)$ the set of such mappings. More generally we make the following definition.

Definition 2.3. Suppose that $\left(A_{N}\right)$ is a sequence such that if $\Omega^{\prime} \subset \subset \Omega$ then 
$A_{N}$ is a linear mapping from $C_{0}^{\infty}\left(\Omega^{\prime}\right)$ to $\mathfrak{D}^{\prime}\left(\Omega^{\prime}\right)$, for large $N$. Let $\left(\phi_{N}\right)$ be a se quence of functions in $C_{0}^{\infty}(\Omega)$ with support in a fixed compact set and satisfying (1.1). Assume now that for every such sequence $\left(\phi_{N}\right)$ there is a sequence $\left(a_{N}\right) \epsilon$ $\tau^{r}(\Omega)$ such that, if $u \in C_{0}^{\infty}(\Omega)$ and $N$ is large, $A_{N}\left(\phi_{N} u\right)(x)=a_{N}(x, D) u(x)$. We then say that $\left(A_{N}\right) \in T^{r}(\Omega)$.

The formula (2.12) extends to define a sequence of kernels $\left(K_{N}(x, y)\right)$ for any sequence $\left(A_{N}\right) \in T^{r}(\Omega)$. We only have to suppose that $\phi_{N}(y)=1$ when $(x, y)$ belongs to the support of $w$. Clearly $T_{0}(\Omega) \subset T^{r}(\Omega)$ for every $r$. We shall now define another subclass of $T^{r}(\Omega)$.

Definition 2.4. A sequence $\left(A_{N}\right) \in T^{r}(\Omega)$ is called properly supported if the corresponding kernels $K_{N}(x, y)$ have support in a set $M \subset \Omega \times \Omega$ such that both projections $M \rightarrow \Omega$ are proper.

Every sequence $\left(A_{N}\right) \in T^{r}(\Omega)$ can now be written as a sum $\left(A_{N}^{\prime}+A_{N}^{\prime \prime}\right)$ where $\left(A_{N}^{\prime}\right)$ is properly supported and $\left(A_{N}^{\prime \prime}\right) \in T_{0}(\Omega)$. In fact, let $M$ be a neighborhood of the diagonal in $\Omega$ such that both projections $M \rightarrow \Omega$ are proper. Choose functions $\chi_{N} \in C^{\infty}(\Omega \times \Omega)$ such that $\chi_{N}(x, y)=1$ in a fixed neighborhood of the diagonal, $\chi_{N}$ has support in $M$ and satisfies (1.1) on compact subsets of $\Omega \times \Omega$. Let $\left(K_{N}\right)$ be the sequence of kernels corresponding to $\left(A_{N}\right)$ and denote by $\left(A_{N}^{\prime \prime}\right)$ the sequence of operators corresponding to $\left(\left(1-\chi_{N}\right) K_{N}\right)$. Then $\left(A_{N}^{\prime \prime}\right) \in T_{0}(\Omega)$ and $\left(A_{N}^{\prime}\right)=\left(A_{N}-A_{N}^{\prime \prime}\right)$ is properly supported.

If $\left(B_{N}\right) \in T^{r}(\Omega)$ is properly supported, it is easy to see that there is a sequence $\left(b_{N}\right) \in \tau^{r}(\Omega)$ such that $\left(B_{N}\right)=\left(b_{N}(x, D)\right)$. Therefore, every sequence $\left(A_{N}\right)$ $\epsilon T^{r}(\Omega)$ has a symbol in the sense of the following definition.

Definition 2.5. The sequence $\left(a_{N}\right) \in \tau^{r}(\Omega)$ is called a symbol of $\left(A_{N}\right) \in T^{r}(\Omega)$ if $\left(A_{N}-a_{N}(x, D)\right) \in T_{0}(\Omega)$.

The symbol of a sequence $\left(A_{N}\right) \in T^{r}(\Omega)$ is uniquely determined modulo $\tau_{0}(\Omega)$. In fact, we have already shown that if the kernels $K_{N}(x, y)$ corresponding to $\left(a_{N}(x, D)\right) \in T_{0}(\Omega)$ vanish when $y$ is outside a fixed compact subset of $\Omega$, then $\left(a_{N}\right) \in \tau_{0}(\Omega)$. In the general case one just has to multiply $K_{N}(x, y)$ with a sequence $\phi_{N}(y)$ satisfying (1.1), with support in a fixed compact set, and apply Theorem 2.1 .

Theorem 2.1 immediately extends to give the symbol of the composition of two sequences $\left(A_{N}\right) \in T^{r}(\Omega)$ and $\left(B_{N}\right) \in T^{s}(\Omega)$, provided that one of them is properly supported. We are now going to study the effect on the symbol, modulo $\tau_{0}(\Omega)$, of transposing the sequence of operators and of making an analytic change of coordinates in $\Omega$. The space $T_{0}(\Omega)$ is invariant under these operations, so we may suppose that the sequence of operators $\left(A_{N}\right)$ is properly supported. Given a properly supported sequence $\left(A_{N}\right) \in T^{r}(\Omega)$ there is a sequence $\left(a_{N}\right) \in \tau^{r}(\Omega)$ such that, if $u \in C_{0}^{\infty}(\Omega)$ and $N$ is large, then 


$$
\begin{aligned}
\left(A_{N} u\right)(x) & =(2 \pi)^{-n} \int e^{i\langle x, \xi\rangle} a_{N}(x, \xi) \hat{u}(\xi) d \xi \\
& =(2 \pi)^{-n} \int\left(\int e^{i(x-y, \xi)} a_{N}(x, \xi) u(y) d y\right) d \xi
\end{aligned}
$$

Since $\left(A_{N}\right)$ is properly supported, there are functions $\chi_{N} \in C^{\infty}(\Omega \times \Omega)$ satisfying (1.1) on compact subsets and having support in a set $M$, for which both projections $M \rightarrow \Omega$ are proper, such that if $a_{N}^{\prime}(x, y, \xi)=\chi_{N}(x, y) a_{N}(x, \xi)$ then

$$
\left(A_{N} u\right)(x)=(2 \pi)^{-n} \int\left(\int e^{i(x-y, \xi)} a_{N}^{\prime}(x, y, \xi) u(y) d y\right) d \xi .
$$

This representation of $\left(A_{N}\right)$ is particularly useful for the study of the transposed sequence and the sequence obtained after a change of variables. Suppose more generally that $b_{N}^{\prime}(x, y, \xi)$ are functions such that for every compact set $L \subset \Omega \times \Omega$ there are positive constants $\delta$ and $C$ such that $b_{N}^{\prime} \in C^{[\delta N]}\left(L \times R^{n}\right)$, when $N \geq$ $\delta^{-2}$, and

$$
\begin{array}{r}
\sup _{x, y \in L}\left|D_{x}^{\alpha} D_{y}^{\beta} D_{\xi}^{\gamma} b_{N}^{\prime}(x, y, \xi)\right| \leq C^{N} N^{|\alpha|+|\beta|}(1+|\xi| / N)^{r-|\gamma|,} \\
\text { when }|\alpha|+|\beta|+|\gamma| \leq \delta N .
\end{array}
$$

Then (2.14) defines a sequence of operators $\left(B_{N}\right)$. We shall show that this sequence still belongs to $T^{r}(\Omega)$. Clearly we may suppose that $b_{N}^{\prime}(x, y, \xi)$ vanishes when $(x, y)$ is outside a set $M \subset \Omega \times \Omega$, such that both projections $M \rightarrow \Omega$ are proper. In fact, every sequence. $\left(B_{N}\right)$ may be written as a sum of a sequence of this type and a sequence in $T_{0}(\Omega)$. Now put $b_{N}(x, \xi)=e^{-i\langle x, \xi)} B_{N}\left(e^{i(x, \xi)}\right)$, i.e.

$$
b_{N}(x, \xi)=(2 \pi)^{-n} \int\left(\int b_{N}^{\prime}(x, x+y, \xi+\eta) e^{-i(y, \eta)} d y\right) d \eta
$$

and operate with $B_{N}$ under the sign of integration in $u(x)=(2 \pi)^{-n} \int e^{i(x, \xi)} u(\xi) d \xi$. This gives that $\left(B_{N} u\right)(x)=b_{N}(x, D) u(x)$, so we have to show that $\left(b_{N}\right) \epsilon$ $\tau^{r}(\Omega)$. Put $d_{N}(x, y, \xi)=b_{N}^{\prime}(x, x+y, \xi)$ and denote by $\hat{d}_{N}$ the Fourier transform of $d_{N}$ with respect to $y$. Then

$$
b_{N}(x, \xi)=(2 \pi)^{-n} \int \hat{d}_{N}(x, \eta, \xi+\eta) d \eta
$$

For sufficiently small $\epsilon$, Taylor expansion gives

where

$$
d_{N}(x, \eta, \xi+\eta)=\sum_{|a| \leq \in N}\left(i D_{\xi}\right)^{a} d_{N}(x, \eta, \xi) \eta^{a} / \alpha !+r_{N}(x, \eta, \xi),
$$

$$
r_{N}(x, \eta, \xi)=\sum_{|\gamma|=[\epsilon N]+1}(i \eta)^{\gamma} \int_{0}^{1} D_{\xi}^{\gamma} \hat{d}_{N}(x, \eta, \xi+t \eta)(1-t)|\gamma|-1 d t / \gamma !
$$

Because of the Fourier inversion formula we have 


$$
b_{N}(x, \xi)=\sum_{|a| \leq \in N}\left(i D_{\xi}\right)^{a} D_{x}^{a} b_{N}^{\prime}(x, y, \xi) /\left.\alpha !\right|_{x=y}+r_{N}(x, \xi) .
$$

It remains to show that $\left(r_{N}(x, \xi)\right)=\left((2 \pi)^{-n} \int r_{N}(x, \eta, \xi) d \eta\right) \in \tau_{0}(\Omega)$. Like in the proof of Theorem 2.1 we consider two cases. When $2|\eta| \leq|\xi|$ we note that (2.15) implies that

$$
\begin{aligned}
\left|\eta^{\gamma} D_{x}^{\alpha} D_{\xi}^{\beta+\gamma} \hat{d}_{N}(x, \eta, \xi+t \eta)\right| / \gamma ! \\
\quad \leq C_{1}^{N}{ }_{N}|a|(1+|\xi+t \eta| / N)^{r-|\gamma|-|\beta|}(1+|\eta|)^{-n-1} N_{N}|\gamma| / \gamma ! \\
\quad \leq C_{2}^{N} N^{N}|a|(1+|\xi| / N)^{r-|\gamma|-|\beta|}(1+|\eta|)^{-n-1} .
\end{aligned}
$$

And when $|\xi| \leq 2|\eta|$ we get from (2.15)

$$
|\xi|^{j}\left|\eta^{\gamma} D_{x}^{\alpha} D_{\xi}^{\beta}+\gamma \hat{d}_{N}(x, \eta, \xi+t \eta)\right| / \gamma ! \leq\left. C^{N}\right|^{|a|+j}(1+|\eta|)^{-n-1} .
$$

It follows immediately from (2.16) and (2.17) that $\left(r_{N}(x, \xi)\right) \in \tau_{0}(\Omega)$ and we have proved that

$$
\left(b_{N}(x, \xi)\right) \sim\left(\sum_{|a| \leq \in N}\left(i D_{\xi}\right)^{a} D_{x}^{a} b_{N}^{\prime}(x, y, \xi) /\left.\alpha !\right|_{x=y}\right) .
$$

From this result one proves the following two theorems in exactly the same way as the corresponding results are proved on pp. 105-109 of [7].

Theorem 2.2. Suppose that $\left(A_{N}\right) \in T^{r}(\Omega)$. Then the sequence of transposed operators $\left({ }^{t} A_{N}\right)$ also belongs to $T^{r}(\Omega)$. If $\left(a_{N}\right),\left({ }^{t} a_{N}\right)$ are symbols of $\left(A_{N}\right)$ and $\left({ }^{t} A_{N}\right)$ respectively, we bave

$$
\left({ }^{t} a_{N}(x, \xi)\right) \sim\left(\sum_{|a| \leq \epsilon N} i|a| D_{x}^{a} D_{\xi}^{a} a_{N}(x,-\xi) / \alpha !\right),
$$

provided that $\epsilon$ is small enough.

If $\kappa: \Omega \rightarrow \Omega$ is an analytic diffeomorphism and $\left(A_{N}\right) \in T^{r}(\Omega)$ we put

$$
\left(A_{N}^{\kappa} u\right)(x)=A_{N}(u \circ \kappa)\left(\kappa^{-1}(x)\right) \text {. }
$$

Theorem 2.3. Suppose that $\left(A_{N}\right) \in T^{r}(\Omega)$ and that $\kappa: \Omega \rightarrow \Omega$ is an analytic diffeomorphism. Then the sequence $\left(A_{N}^{K}\right)$, defined by (2.20), also belongs to $T^{r}(\Omega)$. Denote by $\psi_{a}(x, \xi)$ the following polynomial, of degree $\leq|\alpha| / 2$, in $\xi$ :

$$
\psi_{\alpha}(x, \xi)=D_{y}^{a} \exp \left(\left.i\left\langle_{\kappa}(y)-\kappa(x)-\kappa^{\prime}(x)(y-x), \xi\right\rangle\right|_{x=y^{\circ}} .\right.
$$

If $\left(a_{N}\right),\left(a_{N}^{\kappa}\right)$ are symbols of $\left(A_{N}\right)$ and $\left(A_{N}^{\kappa}\right)$ respectively, we bave 


$$
\left(a_{N}^{\kappa}(\kappa(x), \xi)\right) \sim\left(\sum_{|\alpha| \leq \epsilon N} a_{N}^{(\alpha)}\left(x,{ }^{t} \kappa^{\prime}(x) \xi\right) \psi_{\alpha}(x, \xi) / \alpha !\right)
$$

provided that $\epsilon$ is small enough. Here $a_{N}^{(\alpha)}(x, \eta)=\left(i D_{\eta}\right)^{a} a_{N}(x, \eta)$.

3. Analytic wave front sets and sequences of pseudo-differential operators. For $\left(C^{\infty}\right)$ wave front sets there is, besides a definition corresponding to Definition 1.1, an alternative description of $W F(u)$ based on the elliptic regularity theorem for pseudo-differential operators (see [7] and [8]). We shall now give a similar characterization of $\mathrm{WF}_{a}(u)$ using the sequences of pseudo-differential operators introduced in the previous section.

Definition 3.1. $\left(x_{0}, \xi_{0}\right)$ is called noncharacteristic with respect to $\left(a_{N}\right) \epsilon$ $\tau^{r}(\Omega)$ if and only if there is a conic neighborhood $F$ of $\left(x_{0}, \xi_{0}\right)$ and a decomposition $a_{N}=a_{N}^{r}+a_{N}^{r-1}$, with $\left(a_{N}^{r-1}\right) \in \tau^{r-1}(\Omega)$, such that

$$
\left|a_{N}^{r}(x, \xi)\right| \geq|\xi|^{r} / C, \quad \text { when }(x, \xi) \in F \text { and }|\xi| \geq C N
$$

for some constant $C>0$.

Lemma 3.1. Suppose that $u \in \mathcal{E}^{\prime}(\Omega)$. Then $\left(x_{0}, \xi_{0}\right) \notin \mathbb{W} \mathrm{F}_{a}(u)$ if and only if there are positive constants $\delta$ and $C$ and a sequence $\left(a_{N}\right) \in \tau^{r}(\Omega)$, for some $r$, such that $\left(x_{0}, \xi_{0}\right)$ is noncharacteristic with respect to $\left(a_{N}\right)$ and

$$
\left|D_{x}^{a} a_{N}(x, D) u(x)\right| \leq C^{N}|a|, \text { when }|a| \leq \delta N \text { and } N \geq \delta^{-2} \text {. }
$$

Proof. We shall construct a "local parametrix" $\left(e_{N}(x, D)\right) \in T^{-r}(\Omega)$, such that $\left(e_{N} \circ a_{N}\right)(x, \xi) \sim 1$ in a conic neighborhood $F_{1}$ of $\left(x_{0}, \xi_{0}\right)$ with $\dot{F}_{1} \subset F$. To do this we put $e_{N}^{-r}=1 / a_{N}^{r}$ in $F \cap\{\xi ;|\xi| \geq C N\}$. When $k \leq \delta^{\prime} N,|\xi| \geq C N$ and $(x, \xi) \in F$, we then recursively define $e_{N}^{-r-k}$ by

Finally put

$$
e_{N}^{-r-k}=\sum_{|a|+l=k-j>0}{ }_{i}|a| D_{\xi}^{a} e_{N}^{-r-j} \cdot D_{x}^{a} a_{N}^{r-l} \cdot e_{N}^{-r} .
$$

$$
e_{N}(x, \xi)=\sum_{k \leq \delta^{\prime} N} e^{-r-k}(x, \xi) \cdot \mu_{N}(x, \xi),
$$

for some sequence $\left(\mu_{N}\right) \in \tau^{0}\left(F_{1}, F\right)$, which vanishes when $|\xi| \leq C N$. To check that $\left(e_{N}\right) \in \tau^{-r}(\Omega)$, we have to prove that

$$
\left|D_{x}^{a} D_{\xi}^{\beta} e_{N}^{-r-k}(x, \xi)\right| \leq C_{1}^{N}(1+|\xi| / N)^{-r-k-\left|a_{+} \beta\right|}, \text { when }|\alpha+\beta| \leq \delta^{\prime \prime} N
$$

For $k=0$ we note that $D_{x}^{a} D_{\xi}^{\beta}\left(e_{N}^{-r} \cdot a_{N}^{r}\right)=0$, if $|\alpha+\beta| \neq 0$. Therefore (3.4) follows from Leibniz' formula and (3.3). Choose now sequences $\left(\phi_{N}(x)\right),\left(\psi_{N}(\xi)\right) \epsilon$ $\tau^{0}(\Omega)$ such that $\phi_{N}(x)=1$ in a neighborhood of $x_{0}, \psi_{N}(\xi)=1$ in a conic neighborhood of $\xi_{0}$, when $|\xi| \geq C N$, and $\phi_{N}(x) \psi_{N}(\xi)$ vanishes outside $F_{1}$. Then 


$$
\left(\psi_{N}(D) \phi_{N}(x)\left(e_{N} \circ a_{N}\right)(x, D)-\psi_{N}(D) \phi_{N}(x)\right) \in T_{0}(\Omega)
$$

Because of (3.2), this implies that

$$
\left|D_{x}^{\alpha} \psi_{N}(D) \phi_{N}(x) u(x)\right| \leq C^{N}|a|, \quad \text { when }|a| \leq \delta N \text {. }
$$

If we put $\phi_{N}^{\prime}=\phi_{\left[N, \delta^{-1}\right]+1}$, then $u_{N}(x)=\phi_{N}^{\prime}(x) u(x)$ satisfies (1.3).

For a linear differential operator $P(x, D)$ with analytic coefficients, Lemma 3.1 implies Theorem 1.1. We shall now give two lemmas about the existence of suitable cut-off functions in $\tau^{0}(\Omega)$, which will be used in the proofs of Theorem 1.2 and Theorem 1.3. Suppose that $F$ and $F^{\prime}$ are two open cones in $\Omega \times \dot{R}^{n}$ such that $\dot{F} \subset F^{\prime}$ and let $\left(\mu_{N}\right) \in \tau^{0}\left(F, F^{\prime}\right)$. If $p=\Sigma_{-\infty<k \leq 1} p^{k}$ is an analytic symbol of order 1 in $F^{\prime}$, we put

$$
p_{N}(x, \xi)=\sum_{-N \leq k \leq 1} p^{k}(x, \xi) \mu_{N}(x, \xi) .
$$

Lemma 3.2. Suppose that $p^{1}(x, \xi)$ is real and that $d_{\xi} p^{1}(x, \xi) \neq 0$ in $F^{\prime}$. Let $k$ be a bicharacteristic for $p^{1}$ in $F^{\prime}$. If $F^{\prime}$ is sufficiently small and $F^{\prime \prime}$ is an arbitrary conic neighborbood of $b$, then there is a sequence $\left(a_{N}\right) \in \tau^{0}(\Omega)$ such that $a_{N}(x, \xi)$ vanishes in $F \backslash F^{\prime \prime}$, the points on $b \cap F$ are noncharacteristic with respect to $\left(a_{N}\right)$ and $\left(\left[p_{N}(x, D), a_{N}(x, D)\right]\right) \in T_{0}(\pi(F))$. Here $\pi$ denotes the projection $T^{*}(\Omega) \rightarrow \Omega$.

Proof. We shall choose $a_{N}(x, \xi)$ as a sum $\Sigma_{-N \leq k \leq 0} a_{N}^{k}(x, \xi) \chi_{N}(\xi / N)$, where $a_{N}^{k}$ is homogeneous of degree $k$ and $\chi_{N}(\xi)$ are functions which satisfy (1.1), vanish when $|\xi| \leq C N$ and are equal to 1 when $|\xi| \geq 2 C N$. We want the symbol sequence of $\left(\left[p_{N}(x, D), a_{N}(x, D)\right]\right)$ to be in $\tau_{0}(\pi(F))$. Since $a_{N}(x, \xi)$ will vanish when $\mu_{N}(x, \xi) \neq 1$ and since, in view of Example 2.4 , it is irrelevant how the symbol looks for $|\xi| \leq C N$, it will, because of Theorem 2.1, be sufficient to find solutions $a_{N}^{k}$ to the equations

$$
H_{p}{ }_{1}\left(a_{N}^{k}\right)=\Phi_{k}\left(p^{1}, \cdots, p^{k+1}, a_{N}^{0}, \cdots, a_{N}^{k+1}\right), \quad k \leq \delta^{\prime} N
$$

where

$$
\Phi_{k}\left(p^{1}, \ldots, a_{N}^{k+1}\right)=\sum_{j-|a|=k-l>0} i(|a|-1)\left(D_{\xi}^{a} p^{j} D_{x}^{a} a_{N}^{l}-D_{\xi}^{a} a_{N}^{l} D_{x}^{a} p^{j}\right) / \alpha !
$$

with the following properties:

(3.6) $a_{N}^{k}(x, \xi)$ is homogeneous of degree $k$ and vanishes in $F \backslash F^{\prime \prime}$;

(3.7) $a_{N}^{0}(x, \xi)=1$ in a fixed conic neighborhood of $b \cap F$;

(3.8) $\left|D_{x}^{a} D_{\xi}^{\beta} a_{N}^{k}(x, \xi)\right| \leq\left. C^{N}\right|^{|a+\beta|+k}$, when $|\alpha+\beta|+k \leq \delta N$, on compact subsets of $F^{\prime}$. 
Because of the homogeneity of $a_{N}^{k},(3.8)$ shows that $\left(a_{N}\right) \in \tau^{0}(\Omega)$. Moreover, since $a_{N}^{k}$ satisfies $(3.5)$, we will have $\left(\left[p_{N}(x, D), a_{N}(x, D)\right]\right) \in T_{0}(\pi(F))$. Now $p^{1}(x, \xi)$ is a first order real symbol of principal type. If $F^{\prime}$ is small enough, we can therefore find an analytic change of coordinates $(y, \eta) \mapsto(x(y, \eta), \xi(y, \eta))$ such that $x(y, \eta)$ and $\xi(y, \eta)$ are homogeneous of degree 0 and 1 respectively and $H_{p^{r}}$ is transformed to $\partial / \partial y_{n}$. Denote $a_{N}^{k}(x(y, \eta), \xi(y, \eta))$ by $b_{N}^{k}(y, \eta)$. For $k=0,(3.5)$ reduces to $\partial b_{N}^{0} / \partial y_{n}=0$. If $y^{\prime}=\left(y_{1}, \ldots, y_{n-1}\right)$ we choose $b_{N}^{0}=$ $b_{N}^{0}\left(y^{\prime}, \eta\right)$ independent of $y_{n}$ and so that (3.6)-(3.8) are satisfied by $a_{N}^{0}$. For any function $g(y, \eta)=\Sigma_{0 \leq i} c_{i}\left(y^{\prime}, \eta\right) y_{n}^{i}$, which is analytic in $y_{n}$, we put

$$
(\operatorname{Ig})(y, \eta)=\sum_{0 \leq i} c_{i}\left(y^{\prime}, \eta\right) y_{n}^{i+1} /(i+1)
$$

Denoting $\Phi_{k}\left(p^{1}, \ldots, a_{N}^{k+1}\right)(x(y, \eta), \xi(y, \eta))$ by $g_{N}^{k}(y, \eta)$, we now solve the equations (3.5) recursively by putting $b_{N}^{k}=I g_{N}^{k}$. Clearly the functions $a_{N}^{k}$ obtained in this way, satisfy (3.6) and (3.7). It remains to prove the estimates (3.8). If we put $D=\left(D_{y_{1}}, \cdots, D_{y_{n}}\right)$, it follows from (3.5) that $b_{N}^{k}$ is a sum of less that $C^{k}$ terms $b_{N}^{k}$ of the form

$$
b_{N}^{k}=\left(I \circ\left(B_{m_{1}} D^{a}{ }^{1}\right) \circ I \circ\left(B_{m_{2}} D^{a} 2\right) \circ \cdots \circ I \circ\left(B_{m_{l}} D^{a} l\right)\right)\left(b_{N}^{0}\right),
$$

where $\Sigma_{1 \leq i \leq l}\left(m_{i}+\left|\alpha_{i}\right|\right) \leq k+l \leq 2 k$ and

$$
\sup _{(y, \eta) \in M}\left|D^{\beta} B_{m_{i}}(y, \eta)\right| \leq C^{\left(m_{i}+|\beta|\right)}\left(m_{i}\right) ! \beta ! .
$$

Here $M$ is compact and $C=C(M)$ is independent of $i$ and $k$. In particular each $B_{m_{i}}$ is an analytic function. To prove (3.8), we have to prove that, on compact sets $\left|D^{\gamma} b_{N}^{k}(y, \eta)\right| \leq C^{N}|\gamma|+k$, when $|\gamma|+k \leq \delta N$. Now all derivatives except $\partial / \partial y_{n}$ commute with $I$, and $\partial / \partial y_{n} \circ I$ is the identity. If the differentiations are carried out, then $D^{\gamma} b_{N}^{k}$ may be written as a sum of less than $C^{N}$ terms of the form

$$
v_{N}^{k}=\left(I \circ B_{m_{1}^{\prime}}^{\prime} \circ I \circ B_{m_{2}^{\prime}}^{\prime} \circ \cdots \circ I \circ B_{m_{l^{\prime}}^{\prime}}^{\prime}\right)\left(D^{\beta_{b_{N}}^{0}}\right),
$$

where $|\beta|+\Sigma_{1 \leq i \leq l^{\prime}} m_{i}^{\prime} \leq k+l+|\gamma|$ and the functions $B_{m_{i}^{\prime}}^{\prime}(y, \eta)$ satisfy estimates of the type (3.11). To estimate (3.12), we note that

$$
\left|\left(I \circ f_{1} \circ I \circ f_{2} \circ \cdots \circ I \circ f_{l-1} \circ I\right)\left(f_{l}\right)\right| \leq \prod_{1 \leq i \leq l}\left(\sup _{i}\left|f_{i}\right|\right)\left|y_{n}\right|^{l} / l ! .
$$

Therefore

$$
\left|v_{N}^{k}\right| \leq C^{N}\left(\prod_{1 \leq i \leq l^{\prime}}\left(m_{i}^{\prime} !\right)\right) N^{|\beta|} / l^{\prime} ! \leq C^{N} N^{k+|\gamma|}
$$


This proves (3.9) and completes the proof of Lemma 3.2.

Lemma 3.3. Suppose that $d_{\xi} \operatorname{Re} p^{1}(x, \xi)$ and $d_{\xi} \operatorname{Im} p^{1}(x, \xi)$ are linearly independent and that the Poisson bracket $\left\{\operatorname{Re} p^{1}, \operatorname{Im} p^{1}\right\}(x, \xi)$ vanishes in $F^{\prime}$. Let $b$ be a bicharacteristic strip for $p^{1}$ in $F^{\prime}$. If $F^{\prime}$ is sufficiently small and $F^{\prime \prime}$ is an arbitrary conic neighborbood of $b$, then there is a sequence $\left(a_{N}\right) \in \tau^{0}(\Omega)$ such that $a_{N}$ vanishes in $F \backslash F^{\prime \prime}$, the points on $b \cap F$ are noncharacteristic with respect to $\left(a_{N}\right)$ and $\left(\left[p_{N}(x, D), a_{N}(x, D)\right]\right) \in T_{0}(\pi(F))$.

The proof of this lemma parallels the proof of the preceding one. Because of the Frobenius integrability theorem, there is an analytic change of coordinates $(y, \eta) \mapsto(x(y, \eta), \xi(y, \eta))$ such that $x(y, \eta)$ and $\xi(y, \eta)$ are homogeneous of degree 0 and 1 respectively and $H_{p 1}$ is transformed to $\left(\partial / \partial y_{n}+i \partial / \partial y_{n-1}\right) / 2$. Again we denote $a_{N}^{k}(x(y, \eta), \xi(y, \eta))$ by $b_{N}^{k}(y, \eta) . b_{N}^{0}$ is taken to be independent of $y_{n}$ and $y_{n-1}$ so the right-hand side of (3.5) will in the new coordinates be a convergent power series

$$
g\left(y^{\prime \prime}, z, \bar{z}, \eta\right)=\sum_{0 \leq i} c_{i}\left(y^{\prime \prime}, z, \eta\right) \bar{z}^{i}
$$

where $z=y_{n}+i y_{n-1}, \bar{z}=y_{n}-i y_{n-1}$ and $y^{\prime \prime}=\left(y_{1}, \ldots, y_{n-2}\right)$. Finally the operator $I$ is, in this case, defined by

$$
(\operatorname{Ig})\left(y^{\prime \prime}, z, \bar{z}, \eta\right)=\sum_{0 \leq i} c_{i}\left(y^{\prime \prime}, z, \eta\right) \bar{z}^{i+1} /(i+1)
$$

With these modifications exactly the same proof as for Lemma 3.2 works for Lemma 3.3.

4. An inequality. We start with the following simple lemma:

Lemma 4.1. Suppose that the functions $b_{N}(x, \xi) \in C^{\infty}\left(R^{n} \times R^{n}\right)$ vanish when $x$ is outside a fixed compact set and satisfy

$$
\left|D_{x}^{a} D_{\xi}^{\beta} b_{N}(x, \xi)\right| \leq C_{N}(1+|\xi|)-|\beta|, \quad \text { when }|\alpha| \leq n+1+j,|\beta| \leq j .
$$

Put $B_{N} u(x)=(2 \pi)^{-n} \int e^{i(x, \xi)} b_{N}(x, \xi) \hat{u}(\xi) d \xi$. If (4.1) is satisfied for $j=0$, then $B_{N}$ is a bounded operator on $L^{2}\left(R^{n}\right)$ with norm $\leq M C_{N}$, for some constant $M$ independent of $N$. Moreover, if (4.1) is satisfied with $j=1$ and $b_{N}(x, \xi)$ is real, then $B_{N} \circ \Lambda-\Lambda \circ B_{N}^{*}$ is a bounded operator on $L^{2}\left(R^{n}\right)$ with norm $\leq M C_{N}$. Here $\Lambda=\left(1+|D|^{2}\right)^{1 / 2}$.

Proof.'Parseval's formula gives

$$
\int \overline{v(x)} \cdot B_{N} u(x) d x=(2 \pi)^{-2 n} \iint \overline{\hat{v}(\eta)} \hat{b}_{N}(\eta-\xi, \xi) \hat{u}(\xi) d \xi d \eta \text {. }
$$

Because of (4.1), we have $\left|\hat{b}_{N}(\eta-\xi, \xi)\right| \leq M_{1} C_{N}(1+|\eta-\xi|)^{-n-1}$. Therefore

$$
\left|\int \overline{v(x)} \cdot B_{N} u(x) d x\right| \leq M \cdot C_{N}\|u\| \cdot\|v\| .
$$


This proves the first part of the lemma. For the second part we observe that the adjoint $B_{N}^{*}$ of $B_{N}$ is given by

$$
\widehat{\left(B_{N}^{*} u\right)}(\eta)=\int e^{-i\langle x, \eta)} \bar{b}_{N}(x, \eta) u(x) d x
$$

Thus, if $a_{N}(x, \xi)=b_{N}(x, \xi)\left(1+|\xi|^{2}\right)^{1 / 2}$, we have

$$
\left.\widehat{\left(\Lambda \circ B_{N}^{*} u\right.}\right)(\eta)=(2 \pi)^{-n} \int_{\hat{a}_{N}}(\eta-\xi, \eta) \hat{u}(\xi) d \xi
$$

and

$$
\left.\widehat{\left(B_{N} \circ \Lambda u\right.}\right)(\eta)=(2 \pi)^{-n} \int \hat{a}_{N}(\eta-\xi, \xi) \hat{u}(\xi) d \xi
$$

Now $a_{N}$ is real and, because of (4.1),

$$
\left|\hat{a}_{N}(\eta-\xi, \eta)-\hat{a}_{N}(\eta-\xi, \xi)\right| \leq M_{2} \cdot C_{N}(1+|\xi-\eta|)^{-n-1}
$$

The same argument as for the first part of the lemma now finishes the proof.

Before we state the main result of this section we have to introduce some notation. Let $\Omega$ be a neighborhood of the origin in $R^{n}$ and $\Gamma$ an open cone in $R^{n-1}$. Suppose that $S^{k}\left(x, \dot{\xi}^{\prime}\right), k=1,0,-1, \cdots$, are analytic functions in $\Omega \times \Gamma$, homogeneous of degree $k$, such that, for some constant $C_{0}$ independent of $k$,

$$
\begin{aligned}
& \left|D_{x}^{a} D_{\xi^{\prime}}^{\beta} S^{k}\left(x, \xi^{\prime}\right)\right| \leq C_{0}^{|a+\beta|-k+2}|k| !|\alpha| !|\beta| !\left|\xi^{\prime}\right|^{k-|\beta|}, \\
& \text { when }\left(x, \xi^{\prime}\right) \in \Omega \times \Gamma \text {. }
\end{aligned}
$$

Let $F$ be a closed cone with $\dot{F} \subset \Omega \times \Gamma \times R$ and put $L^{1}(x, \xi)=i \xi_{n}+S^{1}\left(x, \xi^{\prime}\right)$. We shall assume that $L^{1}$ is principally normal in some conic neighborhood $F_{1}$ of $F$, i.e. there exists a function $\lambda(x, \xi)$, analytic in $F_{1}$ and homogeneous of degree zero, such that

$$
i\left\{L^{1}, L^{1}\right\}(x, \xi)=\operatorname{Re}\left(L^{1}(x, \xi) \lambda(x, \xi)\right), \quad \text { when }(x, \xi) \in F_{1} \cdot
$$

Now choose functions $\phi_{N} \in C_{0}^{\infty}(\Omega), \psi_{N} \in C^{\infty}\left(\dot{R}^{n-1}\right)$ and $\chi_{N} \in C^{\infty}\left(R^{n-1}\right)$ such that $\psi_{N}$ is homogeneous. of degree zero and has support in $\Gamma, \phi_{N}(x) \psi_{N}\left(\xi^{\prime}\right)=1$ on $F_{1}, \chi_{N}$ vanishes when $\left|\xi^{\prime}\right| \leq 2 C_{0}$ and equals 1 when $\left|\xi^{\prime}\right| \geq 3 C_{0}$. Put $\rho_{N}\left(x, \xi^{\prime}\right)=\phi_{N}(x) \psi_{N}\left(\xi^{\prime}\right) \chi_{N}\left(\xi^{\prime} / N\right)$. If $\phi_{N}, \psi_{N}$, and $\chi_{N}$ are suitably chosen, we will have

$$
\left|D_{x}^{a} D_{\xi}^{\beta} \rho_{N}\left(x, \xi^{\prime}\right)\right| \leq c^{|a+\beta|+1 N}|\alpha|\left(1+\left|\xi^{\prime}\right| / N\right)-|\beta|, \quad \text { when }|\alpha+\beta| \leq N,
$$

and

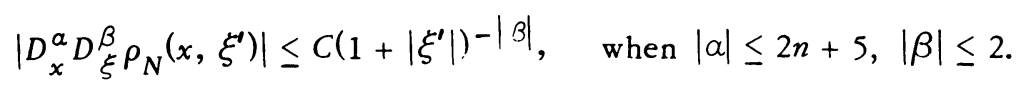


We define

$$
\begin{aligned}
L_{N}(x, \xi) & =i \xi_{n}+S_{N}\left(x, \xi^{\prime}\right), \\
\text { where } S_{N}\left(x, \xi^{\prime}\right) & =\sum_{-N \leq k \leq 1} S^{k}\left(x, \xi^{\prime}\right) \rho_{N}\left(x, \xi^{\prime}\right) .
\end{aligned}
$$

$L_{N}=L_{N}(x, D)=\partial / \partial x_{n}+S_{N}\left(x, D^{\prime}\right)$ is, strictly speaking, not a pseudo-differential operator in $\Omega$. It is the sum of a differential operator in $x_{n}$ and a pseudodifferential operator in $x^{\prime}$ with "coefficients" depending on $x_{n}$. Finally we assume that $\left(a_{N}\right)$ is a sequence in $\tau^{0}(\Omega)$ such that each $a_{N}$ has support in $F$. Put $\Lambda_{N}^{s}=\left(2+\left|D^{\prime}\right|^{2} / N^{2}\right)^{s / 2}, g_{\sigma}\left(x_{n}\right)=\left(\left(x_{n}-\sigma\right)^{2} / 2-\sigma^{2} / 4\right)$ and denote by $\|\cdot\|$ the $L^{2}$-norm in $x^{\prime}$-space.

Theorem 4.1. Let $L_{N}$ and $a_{N}$ be as above. Suppose that the functions $\phi_{N} \in C_{0}^{\infty}(\Omega)$ satisfy (1.1) and put $u_{N}(x)=\phi_{N}(x) a_{N}(x, D) u(x)$. Then there are positive constants $C$ and $\sigma$ such that, if the functions $\phi_{N}(x)$ vanish when $\left|x_{n}\right| \geq \sigma$ and if $N \geq C$, then

$$
\begin{aligned}
& N \int\left\|\Lambda_{N}^{N g}{ }^{\left(x_{n}\right)} u_{N}\right\|^{2} d x_{n} \\
& \quad \leq C \int\left\|\Lambda_{N}^{N g_{\sigma}\left(x_{n}\right)} L_{N} u_{N}\right\|^{2} d x_{n}+C^{N} \int\|u\|^{2} d x_{n}, \quad u \in C_{0}^{\infty}
\end{aligned}
$$

The constant $\sigma$ does only depend on $C_{0}$ and $F$.

Proof. Put $v_{N}(x)=\Lambda_{N}^{N g_{\sigma}(x} n^{\prime} u_{N}(x)$. Then

$$
\begin{aligned}
L_{N} v_{N}=\Lambda_{N}^{N g}{ }^{\left(x_{n}\right)}{ }_{L_{N}} u_{N}+\left(N\left(x_{n}-\sigma\right) \log \Lambda_{N}\right) v_{N}+R_{N} u_{N}, \\
\text { where } R_{N}=\left[S_{N}\left(x, D^{\prime}\right), \Lambda_{N}^{N g} \sigma_{n}^{\left(x_{n}\right)}\right] .
\end{aligned}
$$

Denote by $R_{N}^{\prime}\left(x, \xi^{\prime}\right)$ the sum

$$
\sum_{1 \leq|\alpha| \leq \sigma N} i|\alpha|_{\xi^{\prime}}^{a}\left(2+\left|\xi^{\prime}\right|^{2} / N^{2}\right)^{N g_{\sigma^{\prime}}\left(x_{n}\right) / 2} D_{x^{\prime}}^{\alpha} S_{N}\left(x, \xi^{\prime}\right) / \alpha !
$$

and let $\delta_{N}\left(x, \xi^{\prime}\right)$ be a sequence of functions that satisfies (4.4), (4.5) and vanishes when $\left(x, \xi^{\prime}, \xi_{n}\right) \notin F_{1}$ for all $\xi_{n}$. Suppose also that $\delta_{N}\left(x, \xi^{\prime}\right)=1$ in $F$, when $\left|\xi^{\prime}\right| \geq 2 C_{0} N$. We may then write

$$
R_{N}=R_{N}^{1}+R_{N}^{2}+R_{N}^{3}
$$

where

$$
\begin{aligned}
& R_{N}^{1}\left(x, \xi^{\prime}\right)=\delta_{N}\left(x, \xi^{\prime}\right) R_{N}^{\prime}\left(x, \xi^{\prime}\right), \\
& R_{N}^{2}\left(x, \xi^{\prime}\right)=\left(1-\delta_{N}\left(x, \xi^{\prime}\right)\right) R_{N}^{\prime}\left(x, \xi^{\prime}\right),
\end{aligned}
$$




$$
R_{N}^{3}\left(x, \xi^{\prime}\right)=\int e^{i\left\langle x^{\prime}, \eta^{\prime}\right\rangle} f_{N}\left(\eta^{\prime}, x_{n}, \xi^{\prime}\right) d \eta^{\prime}
$$

and

$$
\begin{aligned}
& f_{N}\left(\eta^{\prime}, x_{n}, \xi^{\prime}\right)=\sum_{|a|=[\sigma N]_{+1}} i|a|\left(\eta^{\prime}\right)^{a} \hat{S}_{N}\left(\eta^{\prime}, x_{n}, \xi^{\prime}\right) \\
& \text { - } \int_{0}^{1} D_{\xi^{\prime}}^{a}\left(2+\left|\xi^{\prime}+t \eta^{\prime}\right|^{2 / N^{2}}\right)^{N g} \sigma_{n}^{\left(x_{n}\right) / 2}(1-t)^{|\alpha|-1} d t / \alpha ! .
\end{aligned}
$$

Here $\hat{S}_{N}$ denotes the Fourier transform of $S_{N}$ with respect to $x^{\prime}$. Now

$$
\begin{array}{r}
\left|D_{\xi^{\prime}}^{a}\left(2+\left|\xi^{\prime}\right|^{2} / N^{2}\right)^{s / 2}\right| \leq(C \sigma)^{|\alpha|}\left(2+\left|\xi^{\prime}\right|^{2} / N^{2}\right)^{(s-|a|) / 2} \\
\text { if }|s| \leq N \sigma \text { and }|a| \leq 2 N \sigma
\end{array}
$$

Therefore, if $\max \left|x_{n}\right| \leq \sigma g_{\sigma}\left(x_{n}\right)=7 \sigma^{2} / 4$ is less than $\sigma$, we immediately get from (4.12) and Lemma 4.1

$$
\int\left\|R_{N}^{3} u_{N}\right\|^{2} d x_{n} \leq C^{N} \int\left\|u_{N}\right\|^{2} d x_{n} \leq C_{1}^{N} \int\|u\|^{2} d x_{n} .
$$

The last inequality follows from Lemma 4.1 applied to $b_{N}(x, D)=\phi_{N}(x) a_{N}(x, D)$. The same estimate holds for the $L^{2}$-norm of $R_{N}^{2} u_{N}$, i.e.,

$$
\int\left\|R_{N}^{2} u_{N}\right\|^{2} d x_{n} \leq C^{N} \int\|u\|^{2} d x_{n} .
$$

In fact, since $R_{N}^{2}\left(x, \xi^{\prime}\right)$ vanishes in the support of $b_{N}(x, \xi)$, we have $R_{N}^{\overline{2}}\left(x, D^{\prime}\right) \circ b_{N}(x, D) u=R_{N}^{4}(x, D) u$, where

$$
\begin{aligned}
R_{N}^{4}(x, \xi)=\sum_{|\gamma|=[\sigma N]+1} i|\gamma| \int e^{i(x, \eta\rangle}\left(\eta^{\gamma} \hat{b}_{N}(\eta, \xi) / \gamma !\right) \\
\cdot\left(\int_{0}^{1} D_{\xi^{\prime}}^{\gamma} R_{N}^{2}\left(x, \xi^{\prime}+t \eta^{\prime}\right)(1-t)|\gamma|-1 d t\right) d y
\end{aligned}
$$

Because $\left|D_{x}^{a} D_{\xi}^{\gamma}, R_{N}^{2}\left(x, \xi^{\prime}\right)\right| \leq C^{N}$ when $|\alpha| \leq n+1$ and $|\gamma|=[\sigma N]+1$, Lemma 4.1 applies to give (4.15). We now put

$$
R_{N}^{5}=R_{N}^{1} \circ \Lambda_{N}^{-N_{g_{\sigma}}\left(x_{n}\right)} \text {. }
$$

Since $S_{N}\left(x, \xi^{\prime}\right)=\Sigma_{-N \leq k \leq 1} S^{k}\left(x, \xi^{\prime}\right) \chi_{N}\left(\xi^{\prime} / N\right)$ in the support of $\delta_{N}\left(x, \xi^{\prime}\right)$ and $\chi_{N}\left(\xi^{\prime}\right)$ vanishes when $\left|\xi^{\prime}\right| \leq 2 C_{0}$, it follows from (4.2) and (4.13) that

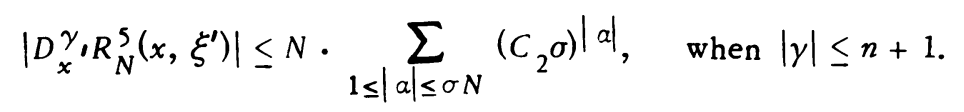

Here the constant $C_{2}$ only depends on $C_{0}$ and the constant $C$ in (4.13). If $\sigma$ is small enough, we get from (4.18) and Lemma 4.1

$$
\int\left\|R_{N}^{5} v_{N}\right\|^{2} d x_{n} \leq C \cdot \sigma \cdot N \int\left\|v_{N}\right\|^{2} d x_{n} .
$$


In view of (4.8), (4.9), (4.14), (4.15), (4.17), and (4.19) it will be sufficient to prove

$$
N \int\left\|v_{N}\right\|^{2} d x_{n} \leq C_{3} \int\left\|L_{N} v_{N}-\left(N\left(x_{n}-\sigma\right) \log \Lambda_{N}\right) v_{N}\right\|^{2} d x_{n}+C^{N} \int\|u\|^{2} d x_{n},
$$

for some constant $C_{3}$ which stays bounded as $\sigma$ tends to zero. Put $S_{N}=A_{N}{ }^{+}$ $i B_{N}$, where $A_{N}$ and $B_{N}$ are real and denote by $($,$) the scalar product in L_{x}^{2}$. Then

$$
\begin{aligned}
\int \| L_{N} v_{N}-( & \left.\left(x_{n}-\sigma\right) \log \Lambda_{N}\right) v_{N} \|^{2} d x_{n} \\
= & \int\left\|\partial v_{N} / \partial x_{n}+i B_{N} v_{N}\right\|^{2} d x_{n} \\
& +\int\left\|A_{N} v_{N}-\left(N\left(x_{n}-\sigma\right) \log \Lambda_{N}\right) v_{N}\right\|^{2} d x_{n}+I_{N}^{1}+I_{N}^{2}+I_{N}^{3},
\end{aligned}
$$

where

$$
\begin{aligned}
I_{N}^{1} & =-2 \operatorname{Re} \int\left(\partial v_{N} / \partial x_{n},\left(N\left(x_{n}-\sigma\right) \log \Lambda_{N}\right) v_{N}\right) d x_{n} \\
& =N \int\left(v_{N},\left(\log \Lambda_{N}\right) v_{N}\right) d x_{n}, \\
I_{N}^{2} & =-2 \operatorname{Re} \int\left(i B_{N} v_{N},\left(N\left(x_{n}-\sigma\right) \log \Lambda_{N}\right) v_{N}\right) d x_{n}, \quad \text { and } \\
I_{N}^{3} & =2 \operatorname{Re} \int\left(\partial v_{N} / \partial x_{n}+i B_{N} v_{N}, A_{N} v_{N}\right) d x_{n} .
\end{aligned}
$$

If we denote $\left(\log \Lambda_{N}\right)^{1 / 2} v_{N}$ by $w_{N}$, we have

$2 \operatorname{Re}\left(i B_{N} v_{N},\left(\log \Lambda_{N}\right) v_{n}\right)=2 \operatorname{Re}\left(i\left[\left(\log \Lambda_{N}\right)^{1 / 2}, B_{N}\right] v_{N}, w_{N}\right)+\left(i\left(B_{N}-B_{N}^{*}\right) w_{N}, w_{N}\right)$.

Because of (4.2), (4.5), (4.6), and the fact that $\chi_{N}\left(\xi^{\prime}\right)$ vanishes when $\left|\xi^{\prime}\right| \leq$ $2 C_{0}$, it follows from Lemma 4.1 that $\left\|\left[\left(\log v_{N}\right)^{1 / 2}, B_{N}\right] v_{N}\right\| \leq C,\left\|v_{N}\right\|$ and $\left\|\left(B_{N}-B_{N}^{*}\right) w_{N}\right\| \leq C\left\|w_{N}\right\|$. Since $\left\|v_{N}\right\| \leq 2 \cdot(\log 2)^{-1 / 2} \cdot\left\|w_{N}\right\|$, we get

$$
\left|I_{N}^{2}\right| \leq C_{4} \cdot \sigma \cdot N \int\left\|w_{N}\right\|^{2} d x_{n} .
$$

To estimate $I_{N}^{3}$, we observe that

$$
\begin{aligned}
I_{N}^{3}= & \int\left(\partial v_{N} / \partial x_{n}+i B_{N} v_{N},\left(A_{N}-A_{N}^{*}\right) v_{N}\right) d x_{n} \\
& +\int\left(\left[A_{N}, \partial / \partial x_{n}+i B_{N}\right] v_{N}, v_{N}\right) d x_{n} .
\end{aligned}
$$

Let now $\nu_{N}(x, \xi)$ be a sequence of functions which satisfy (4.4) and (4.5), with $\xi^{\prime}$ replaced by $\xi$. Suppose also that $\nu_{N}$ has support in $F_{1}$ and equals 1 in $F$, when $|\xi| \geq 2 C_{0} N$. We then write

$$
\left[A_{N}, \partial / \partial x_{n}+i B_{N}\right]=E_{N}^{1}+E_{N}^{2}+E_{N}^{3},
$$

where 


$$
\begin{aligned}
E_{N}^{1}(x, \xi)= & \nu_{N}(x, \xi)\left\{\xi_{n}+B_{N}\left(x, \xi^{\prime}\right), A_{N}\left(x, \xi^{\prime}\right)\right\}, \\
E_{N}^{2}(x, \xi)= & \left(1-\nu_{N}(x, \xi)\right)\left\{\xi_{n}+B_{N}\left(x, \xi^{\prime}\right), A_{N}\left(x, \xi^{\prime}\right)\right\}, \\
E_{N}^{3}(x, \xi)= & \sum_{|\gamma|=2} \int e^{i\left(x^{\prime}, \eta^{\prime}\right)}\left(\eta^{\prime}\right)^{\gamma}\left(\int_{0}^{1} f_{\gamma^{\prime}}\left(x, \eta^{\prime}, \xi^{\prime}, t\right)(1-t) d t\right) d \eta^{\prime} / 2, \text { and } \\
f_{\gamma}\left(x, \eta^{\prime}, \xi^{\prime}, t\right)= & \hat{A}_{N}\left(\eta^{\prime}, x_{n}, \xi^{\prime}\right) D_{\xi^{\prime}}^{\gamma} B_{N}\left(x, \xi^{\prime}+t \eta^{\prime}\right) \\
& -\hat{B}_{N}\left(\eta^{\prime}, x_{n}, \xi^{\prime}\right) D_{\xi^{\prime}}^{\gamma} A_{N}\left(x, \xi^{\prime}+t \eta^{\prime}\right) .
\end{aligned}
$$

Since $\chi_{N}\left(\xi^{\prime}\right)$ vanishes when $\left|\xi^{\prime}\right| \leq 2 C_{0}$, (4.2), (4.5), and (4.6) imply that $\left|D_{x^{\prime}}^{a} E_{N}^{3}\left(x, \xi^{\prime}\right)\right| \leq C$ when $|\alpha| \leq n+1$. Thus Lemma 4.1 gives

$$
\left\|E_{N}^{3} v_{N}\right\| \leq\left\|v_{N}\right\|
$$

Moreover, because of (4.3), there are functions $\mu_{1}$ and $\mu_{2}$ homogeneous of degree zero and analytic in a neighborhood of the support of $\nu_{N}$ such that

$$
\left\{\xi_{n}+B_{N}^{1}\left(x, \xi^{\prime}\right), A_{N}^{1}\left(x, \xi^{\prime}\right)\right\}=\mu_{1}(x, \xi)\left(i \xi_{n}+i B_{N}^{1}\left(x, \xi^{\prime}\right)\right)+\mu_{2}(x, \xi) A_{N}^{1}\left(x, \xi^{\prime}\right.
$$

Put $\nu_{N}^{1}=\nu_{N} \cdot \mu_{1}$ and $\nu_{N}^{2}=\nu_{N} \cdot \mu_{2}$. Then

$$
E_{N}^{1}=\nu_{N}^{1}(x, D) \circ\left(\partial / \partial x_{n}+i B_{N}\right)+\nu_{N}^{2}(x, D) \circ A_{N}+E_{N}^{4},
$$

where

$$
\int\left\|E_{N}^{4} v_{N}\right\|^{2} d x_{n} \leq C \int\left\|v_{N}\right\|^{2} d x_{n}
$$

We now have

$$
\begin{aligned}
\int\left(\left[A_{N}, \partial / \partial_{n}+i B_{N}\right] v_{N}, v_{N}\right) d x_{n} \\
=\int\left(\nu_{N}^{1}(x, D) \circ\left(\partial / \partial x_{n}+i B_{N}\right) v_{N^{\prime}}, v_{N}\right) d x_{n} \\
+\int\left(\nu_{N}^{2}(x, D) \circ\left(A_{N}-N\left(x_{n}-\sigma\right) \log \Lambda_{N}\right) v_{N^{\prime}} v_{N}\right) d x_{n} \\
+N \int\left(\left[v_{N}^{2}(x, D),\left(\log \Lambda_{N}\right)^{1 / 2}\right]\left(x_{n}-\sigma\right) w_{N^{\prime}} v_{N}\right) d x_{n} \\
+N \int\left(\nu_{N}^{2}(x, D)\left(x_{n}-\sigma\right) w_{N^{\prime}}, w_{N}\right) d x_{n}+\sum_{i=2}^{4}\left(E_{N}^{i} v_{N^{\prime}} v_{N}\right)
\end{aligned}
$$

Exactly as for (4.15) it follows that

$$
\int\left\|E_{N}^{2} v_{N}\right\|^{2} d x_{n} \leq C^{N} \int\|u\|^{2} d x_{n}
$$

Since $\left(A_{N}-A_{N}^{*}\right), \nu^{1}(x, D),\left[\nu^{2}(x, D),\left(\log \Lambda_{N}\right)^{1 / 2}\right], E_{N}^{3}$ and $E_{N}^{4}$ are bounded 
operators on $L_{x}^{2}$, it follows from (4.24), (4.29), and (4.30) that, for any $\epsilon>0$,

$$
\left|I_{N}^{3}\right| \leq C_{5}\left(\epsilon \int\left\|\partial v_{N} / \partial x_{n}+i B_{N} v_{N}\right\|^{2} d x_{n}+\epsilon \int\left\|A_{N} v_{N}-\left(N\left(x_{n}-\sigma\right) \log \Lambda_{N}\right) v_{N}\right\|^{2} d x_{n}\right.
$$

$$
\left.+\epsilon^{-1} \int\left\|v_{N}\right\|^{2} d x_{n}+N \cdot \sigma \int\left\|w_{N}\right\|^{2} d x_{n}\right)
$$

$$
+C^{N} \int\|u\|^{2} d x n^{\circ}
$$

Here $C_{5}$ only depends on $C_{0}$ and $F$, while $C$ depends on $b_{N}(x, D)=$ $\phi_{N}(x) a_{N}(x, D)$ and thus on $\sigma$. To prove (4.20) it!now only remains to combine (4.21), (4.22), (4.23), and (4.31). First choose $\epsilon$ so that $C_{5} \cdot \epsilon \leq 1$. Then the first two terms in (4.31) are absorbed by the first two terms in (4.21). After that, choose $\sigma$ so that $2\left(C_{4}+C_{5}\right) \cdot \sigma \leq 1$. Then, if $N$ is large enough,

$$
\begin{aligned}
I_{N}^{1}-C_{3} \epsilon^{-1} \int\left\|v_{N}\right\|^{2} d x_{n}-N\left(C_{4}+C_{5}\right) \sigma \int\left\|w_{N}\right\|^{2} d x_{n} \\
\geq C_{6} N \int\left\|w_{N}\right\|^{2} d x_{{ }_{u}}-C^{N} \int\|u\|^{2} d x_{n} .
\end{aligned}
$$

Since $\left\|v_{n}\right\| \leq 2(\log 2)^{-1 / 2}\left\|w_{N}\right\|$, this completes the proof.

Remark 4.1. We have actually proved a slightly stronger inequality that (4.7). On the left hand side $\Lambda_{N}^{N g_{\sigma}{ }^{\left(x_{n}\right)}}$ could be multiplied by $\left(\log \Lambda_{N}\right)^{1 / 2}$.

Remark 4.2. The proof of Theorem 4.1 is modelled on the proof of Théorème 1 in [12]. Except for technicalities, the main difference is that the norm of $R_{N}^{5}$ has to be estimated as in (4.19).

Remark 4. 3. It was assumed in Theorem 4.1 that $u \in C_{0}^{\infty}$. However, the proof works without change for any $u \in C_{0}$ such that $a_{N}(x, D) u \in C_{0}^{[\delta N]}(\Omega)$ for some $\delta>\max \left|x_{n}\right| \leq \sigma g_{\sigma}\left(x_{n}\right)=7 \sigma^{2} / 4$.

5. Completion of the proofs of Theorems 1.2-1.4. The proofs of the three theorems are similar but, since there are some differences, we give them one by one.

Proof of Theorem 1.2. Clearly it will be sufficient to prove the following "semilocal" statement. Suppose that $\left(x_{0}, \xi_{0}\right) \in N_{1}\left(P_{m}\right) \backslash \mathbb{W F} a_{a}(u)$ and denote by $b$ the bicharacteristic strip through $\left(x_{0}, \xi_{0}\right)$. Then there is a closed conic neighborhood $F$ of $\left(x_{0}, \xi_{0}\right)$ such that if $b \cap F \cap \mathrm{CWF}_{a}(u) \neq \varnothing$ then $k \cap F \cap \mathbb{W F}_{a}(u)=\varnothing$. We shall prove that there is a positive constant $\gamma$, independent of $\left(\bar{x}_{0}, \bar{\xi}_{0}\right) \epsilon$ $F \cap k \cap\{(x, \xi) ;|\xi|=1\}$, such that if $\left(\bar{x}_{0}, \bar{\xi}_{0}\right) \notin \mathbb{W F}_{a}(u)$ then $b \cap B_{\gamma}\left(\bar{x}_{0}, \bar{\xi}_{0}\right) \cap$ $\mathbb{W F}_{\boldsymbol{a}}(u)=\varnothing$. Here $B_{\gamma}\left(\bar{x}_{0}, \xi_{0}\right)=\left\{(\bar{x}, \bar{\xi}) ;\left|\left(\bar{x}-x_{0}, \xi-\xi_{0}\right)\right|<\gamma\right\}$. Since $d_{\xi} P_{m}(x, \xi) \neq 0$ in $N_{1}\left(P_{m}\right)$, we may assume that $\partial P_{m} / \partial \xi_{n} \neq 0$ at $\left(x_{0}, \xi_{0}\right)$. According to a factorization lemma of Hörmander [9, Proposition 6.1] there is a closed conic neighborhood $F_{1}$ of $\left(x_{0}, \xi_{0}\right)$ such that

$$
P(x, \xi)=Q(x, \xi) \circ\left(i \xi_{n}+S\left(x, \xi^{\prime}\right)\right),
$$


in a conic neighborhood of $\dot{F}_{1}$. Here $S\left(x, \xi^{\prime}\right)$ is an analytic symbol in $\left(x, \xi^{\prime}\right)$ and

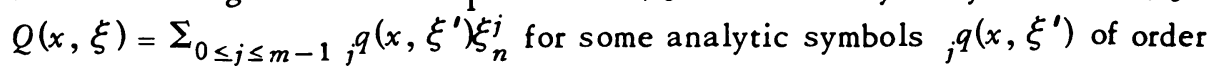
$m-1-j$. The right-hand side of (5.1) stands for the formal composition of the symbols. Suppose that $\dot{\bar{F}} \subset F_{1}$ and that $\left(\bar{x}_{0}, \bar{\xi}_{0}\right) \in b \cap F \cap \mathrm{CWF}_{a}(u)$. Then $(U \times \Gamma) \cap \mathbb{W F}_{a}(u)=\varnothing$, for some conic neighborhood $U \times \Gamma$ of $\left(\bar{x}_{0}, \bar{\xi}_{0}\right)$. We may assume that $U$ is of the form $U^{\prime} \times I$, where $I=\left\{x_{n} ;\left|x_{n}-\bar{x}_{0, n}\right|<\epsilon\right\}$. In view of Lemma 3.2, there is a sequence $\left(a_{N}\right) \in \tau^{0}(\Omega)$ such that supp $a_{N} \subset F_{1}$, (supp $\left.a_{N}\right) \cap$ $\left(R^{n-1} \times I \times \dot{R}^{n}\right) \subset U \times \Gamma$, the points on $b \cap F$ are noncharacteristic with respect to $\left(a_{N}\right)$ and

$$
\left(\left[\Lambda^{1-m} \circ P(x, D), a_{N}(x, D)\right]\right) \in T_{0}(\pi(F)) .
$$

As before, $\pi$ denotes the projection $T^{*}(\Omega) \rightarrow \Omega$ and $\Lambda^{k}=\left(1+|D|^{2}\right)^{k / 2}$. Choose functions $\rho_{N}\left(x, \xi^{\prime}\right)$ satisfying (4.4) and (4.5) with support in a set where $S\left(x, \xi^{\prime}\right)$ and ${ }_{j} q\left(x, \xi^{\prime}\right)$ are defined such that $\rho_{N}\left(x, \xi^{\prime}\right)=1$ in $F_{1}$ when $\left|\xi^{\prime}\right| \geq C N$. Put

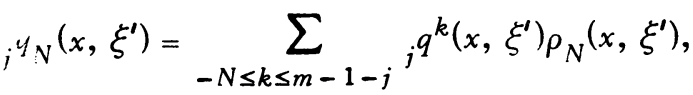

$$
\begin{aligned}
& Q_{N}(x, \xi)=\sum_{0 \leq j \leq m-2}{ }_{j} q_{N}\left(x, \xi^{\prime}\right) \xi_{n}^{j}, \\
& S_{N}\left(x, \xi^{\prime}\right)=\sum_{-N \leq k \leq 1} S^{k}\left(x, \xi^{\prime}\right) \rho_{N}\left(x, \xi^{\prime}\right) \text { and } L_{N}(x, \xi)=i \xi_{n}+S_{N}\left(x, \xi^{\prime}\right) \text {. }
\end{aligned}
$$

Since $\rho_{N}=1$ in $F_{1}$, when $|\xi| \geq C N$, it follows from (5.1) and (5.2) that

$$
\left(Q_{N}(x, D) \circ L_{N}(x, D) \circ a_{N}(x, D)-\Lambda^{m-1} \circ a_{N} \circ \Lambda^{1-m} \circ P(x, D)\right) \in T_{0}(\pi(F))
$$

We may assume that $F_{1} \cap W_{a}(u)=\varnothing$ and that the points in $F_{1}$ are noncharacteristic with respect to $\left(Q_{N}\right)$. Then it follows from (5.3) and Lemma 3.1 that

$$
\left|D_{x}^{\sigma} L_{N}(x, D) \circ a_{N}(x, D) \mathcal{u}(x)\right| \leq C^{N} N|a|, \quad \text { when }|a| \leq \delta N \text { and } x \in \pi(F) \text {. }
$$

Moreover, since $\left(\operatorname{supp} a_{N}\right) \cap\left(R^{n-1} \times I \times \dot{R}^{n}\right) \subset U \times \Gamma$, we have

$$
\left|D_{x}^{a} a_{N}(x, D) u(x)\right| \leq C^{N}|x|,
$$

$$
\text { when }|a| \leq \delta N \text { and } x \notin \pi\left(\operatorname{supp} a_{N}\right) \cap\left(R^{n-1} \times I\right) \text {. }
$$

We are now in the position to apply Theorem 4.1. First of all it is clear that we may suppose that $\bar{x}_{0}=0$. Then $I=\left\{x_{n} ;\left|x_{n}\right|<\epsilon\right\}$. Let $\sigma$ be the constant in Theorem 4.1 and let the functions $\phi_{N}\left(x_{n}\right)$ satisfy $(1.1)$, vanish outside $-\epsilon / 2 \leq$ $x_{n} \leq \sigma$ and be equal to 1 when $0 \leq x_{n} \leq \sigma / 2$. Put $b_{N}(x, \xi)=\phi_{N}\left(x_{n}\right) a_{N}(x, \xi)$. 
Then all assumptions in Theorem 4.1 are fulfilled, except that we do not know that $u \in C_{0}^{\infty}(\Omega)$. However, it follows from the results of [5] that $u_{N}=b_{N}(x, D) u \in$ $C_{0}^{\left[\delta^{\prime} N\right]}(\Omega)$. In fact this, and the corresponding resuits needed for Theorem 1.3 and Theorem 1.4, could be proved directly using the methods of this paper. If $\phi \in C_{0}^{\infty}$ and equals 1 in $\pi\left(F_{1}\right)$, then $v=\phi \Lambda^{-2 k} \phi u \in C_{0}$, if $k$ is large enough, and $P \circ \Lambda^{2 k} v=f$ in $\pi\left(F_{1}\right)$. Therefore we may assume that $u \in C_{0}$ and Remark 4.3 applies. If supp $a_{N}$ is close enough to $b$, it follows from (5.5) that (5.4), with $a_{N}$ replaced by $b_{N}$, is valid when $x_{n} \leq \sigma / 2$. Since $g_{\sigma}\left(x_{n}\right) \leq-\sigma^{2} / 8$ when $\sigma / 2 \leq$ $x_{n} \leq \sigma$, it follows that the right-hand side of (4.7) is bounded by $C^{N}$. Thus

$$
\int_{0 \leq x_{n} \leq \sigma / 4}\left\|\Lambda_{N}^{\lambda \cdot N} u_{N}\right\|^{2} d x_{n} \leq C^{N}, \quad \text { where } \lambda=\min _{0 \leq x_{n} \leq \sigma / 4} g_{\sigma}\left(x_{n}\right)=\sigma^{2} / 32 \text {. }
$$

Because of Euler's identity, $\left\langle\left(d_{\xi} P_{m}\right)\left(x_{0}, \xi_{0}\right), \xi_{0}\right\rangle=m P_{m}\left(x_{0}, \xi_{0}\right)=0$. We may therefore assume that $\left|\xi_{n}\right| \leq \epsilon\left|\xi^{\prime}\right|$ in the support of $b_{N}$ and it follows from (5.6) together with Sobolev's embedding theorem that

$$
\left|D_{x}^{a} a_{N}(x, D) u(x)\right| \leq C^{N}|a| \text {, when }|a| \leq \lambda N \text { and } 0 \leq x_{n} \leq \sigma / 8 \text {. }
$$

Lemma 3.1 now gives that $\left\{(x, \xi) \in b ; 0 \leq x_{n} \leq \sigma / 8\right\} \cap W_{\boldsymbol{a}}(u)=\varnothing$. It follows in the same way that the points on $b$, with $-\sigma_{i}^{\prime} 8 \leq x_{n} \leq 0$, are in the complement of WF $a_{a}(u)$. Since $\sigma$ does not depend on $\bar{x}_{0}$, this completes the proof of Theorem 1.2.

Proof of Theorem 1.3. Let $\left(x_{0}, \xi_{0}\right) \notin N_{2}\left(P_{m}\right) \backslash \mathbb{W} F_{a}(u)$. Then there is an analytic function $\lambda(x, \xi)$ homogeneous of degree $m-1$, in $\xi$, such that

$$
\left\{P_{m}, \bar{P}_{m}\right\}(x, \xi)=\lambda(x, \xi) P_{m}(x, \xi)-\bar{\lambda}(x, \xi) \bar{P}_{m}(x, \xi),
$$

in a conic neighborhood of $\left(x_{0}, \xi_{0}\right)$. We shall multiply $P_{m}$ with a nonvanishing analytic function $g(x, \xi)$ homogeneous of degree $1-m$ in $\xi$, such that

$$
\left\{g P_{m}, \overline{g P}_{m}\right\}(x, \xi)=0
$$

in a full conic neighborhood of $\left(x_{0}, \xi_{0}\right)$. Because of (5.8), we have

$$
\left\{g P_{m}, \overline{g P}_{m}\right\}=2 i \operatorname{Im}\left(\bar{P}_{m}\left(g\left\{P_{m}, \bar{g}\right\}-\bar{\lambda} g \bar{g}+P_{m}\{g, \bar{g}\} / 2\right)\right) \text {. }
$$

It will therefore be sufficient to solve the following nonlinear equation

$$
\left\{P_{m}, \bar{g}\right\}-\overline{\lambda g}+P_{m}\{g, \bar{g}\} / 2 g=0 .
$$

Since the last term vanishes at $\left(x_{0}, \xi_{0}\right)$, we can employ the Cauchy-Kovalevsky Theorem and solve $(5.10)$ in a conic neighborhood of $\left(x_{0}, \xi_{0}\right)$ with data given on any hyperplane with normal $N$, provided that $\left\langle\left(d_{\xi} P_{m}\right)\left(x_{0}, \xi_{0}\right), N\right\rangle \neq 0$. Moreover it follows directly from the uniqueness of the solution that, if we give data homogeneous of degree $k$, then $g$ is homogeneous of degree $k$. Denote by $b$, the 
(2-dimensional) bicharacteristic strip through $\left(x_{0}, \xi_{0}\right)$. Since $\left(x_{0}, \xi_{0}\right) \in N_{2}\left(P_{m}\right)$, we may suppose that there is an open conic neighborhood $F_{1}$ of $\left(x_{0}, \xi_{0}\right)$ such that

$$
\partial P_{m}(x, \xi) / \partial \xi_{k} \neq 0, \quad \text { when } k=n-1, n \text { and }(x, \xi) \in F_{1} .
$$

We shall also assume that (5.9) is satisfied in a conic neighborhood $F_{1}^{\prime}$ of $\dot{\bar{F}}_{1}$. For some $\left(\mu_{N}\right) \in \tau^{0}\left(F_{1}, F_{1}^{\prime}\right)$ we put $g_{N}(x, \xi)=g(x, \xi) \cdot \mu_{N}(x, \xi)$. If $F_{1}^{\prime} \cap$ $\mathbb{W F}(P u)=\varnothing$, then

$$
\left|D_{x}^{a} g_{N}(x, D) \circ P(x, D) u(x)\right| \leq C^{N}|a|, \quad \text { when }|a| \leq \delta N .
$$

Let $F$ and $F_{2}$ be open conic neighborhoods of $\left(x_{0}, \xi_{0}\right)$ such that the $\bar{F} \subset F_{2} \subset$ $\dot{\bar{F}}_{2} \subset F_{1}$ and denote by $F_{3}$ any conic neighborhood of $b$. According to Lemma 3.3 there is a sequence $\left(a_{N}\right) \subset \tau^{0}(\Omega)$ such that supp $a_{N} \subset F_{1} \cap F_{3}$, the points on $k \cap$ $F_{2}$ are noncharacteristic with respect to $\left(a_{N}\right)$ and

$$
\left(\left[g_{N}(x, D) \circ P(x, D), a_{N}(x, D)\right]\right) \in T_{0}\left(\pi\left(F_{2}\right)\right) .
$$

Since we may assume that the points in supp $a_{N}$ are noncharacteristic $w$ ith respect to $\left(g_{N}\right)$, it follows from (5.12) and (5.13) that

$$
\left|D_{x}^{a} P(x, D) \circ a_{N}(x, D) u(x)\right| \leq C^{N}|a|, \quad \text { when }|a| \leq \delta N \text { and } x \in \pi\left(F_{2}\right) .
$$

To prove Theorem 1.3, we shall prove that there is a constant $\gamma>0$, independent of $\left(\dot{\bar{x}}_{0}, \bar{\xi}_{0}\right) \in F \cap k \cap\{(x, \xi) ;|\xi|=1\}$ such that if $\left(\bar{x}_{0}, \bar{\xi}_{0}\right) \notin \mathbb{W} \mathrm{F}_{a}(u)$ then

$$
\boldsymbol{k} \cap B_{\gamma}\left(\bar{x}_{0}, \bar{\xi}_{0}\right) \cap \mathbb{W F}_{a}(u)=\varnothing \text {. }
$$

In fact we shall prove that $(5.15)$ is true for any $\gamma$ such that $B_{\gamma}\left(\bar{x}_{0}, \bar{\xi}_{0}\right) \subset F_{2}$. Suppose that $B_{\gamma}\left(\bar{x}_{0}, \bar{\xi}_{0}\right)$ has distance $\geq \epsilon>0$ to $C_{F_{2}}$ and denote by $S_{\gamma}$ the boundary of $B_{\gamma}$. We shall then prove that there is a constant $\epsilon^{\prime}>0$, independent of $\gamma$, such that if $(\bar{x}, \bar{\xi}) \notin k \cap S_{\gamma}\left(\bar{x}_{0}, \bar{\xi}_{0}\right)$ then $b \cap B_{\epsilon^{\prime}}(\bar{x}, \bar{\xi}) \cap \mathbb{W F}(u)=\varnothing$. Because of (5.11) the normal of $S_{\gamma}\left(\bar{x}_{0}, \bar{\xi}_{0}\right)$ at $(\bar{x}, \bar{\xi})$ is not perpendicular to the $\left(x_{n-1}, x_{n}\right)$-plane. Suppose that it is not perpendicular to the $x_{n}$-axis and that $\bar{x}=0$. Then there are constants $\epsilon^{\prime \prime}$ and $M$ such that $\epsilon^{\prime \prime}$ depends on $F_{3}$ and tends to zero as the neighborhood $F_{3}$ of $b$ becomes smaller, $M$ depends just on $B_{\gamma}\left(\bar{x}_{0}, \bar{\xi}_{0}\right)$ and

$$
D_{x}^{a} a_{N}(x, D) u(x)\left|\leq C^{N^{\prime}}\right| a \mid
$$

$$
\text { when }|a| \leq \delta N \text { and }-1 / M \leq x_{n} \leq-\epsilon^{\prime \prime}-M\left|x^{\prime}\right|^{2} \text {. }
$$

We now make the following change of coordinates:

$$
y_{n}=x_{n}+2 M\left|x^{\prime}\right|^{2}, \quad y^{\prime}=x^{\prime} .
$$


(5.16) is then transformed to

$$
\left|D_{y}^{a} a_{N}(y, D) y(y)\right| \leq C^{N} N^{|a|}, \quad \text { when }|a| \leq \delta N \text { and } y \in G\left(\epsilon^{\prime \prime}, M\right) \text {. }
$$

Here $G\left(\epsilon^{\prime \prime}, M\right)=\left\{y ;-1 / M+2 M\left|y^{\prime}\right|^{2} \leq y{ }_{n} \leq-\epsilon^{\prime \prime}+M\left|y^{\prime}\right|^{2}\right\}$ and we have kept the notation $a_{N}$ for the operator in the new coordinates. The rest of the proof now parallels the proof of Theorem 1.2. By means of the factorization lemma of Hörmander, which works also in the complex case, we obtain an operator $L_{N}(y, D)=$ $\partial / \partial y_{n}+S_{N}\left(y, D^{\prime}\right)$ such that

$$
\left|D_{y}^{a} L_{N}(y, D) \circ a_{N}(y, D) u(y)\right| \leq C^{N}|a|, \quad \text { when }|a| \leq \delta N
$$

and $y$ belongs to a fixed neighborhood of the origin. The results of [5] give that $a_{N}(y, D) u \in C_{0}^{\left[\delta^{\prime} N\right]}$ and since the constant $\sigma$ in Theorem 4.1 does not depend on $\bar{x}$, it follows from Theorem 4.1 that $(5.18)$ is actually satisfied in a neighborhood of $y=0$. Observe that, because of (5.8), $L_{N}$ satisfies the hypothesis in Theorem 4.1. Theorem 1.3 now follows from Lemma 3.1.

Proof of Theorem 1.4. Suppose that $\left(x_{0}, \xi_{0}\right) \in N_{1}\left(P_{m}\right) \backslash W F_{a}(P u)$. Because of The orem 1.3, we just have to consider the case when the bicharacteristic strip $b$ through $\left(x_{0}, \xi_{0}\right)$ is 1 -dimensional. Let $U_{0} \times \Gamma_{0}$ be a conic neighborhood of $\left(x_{0}, \xi_{0}\right)$ such that $Z\left(P_{m}\right) \cap\left(\bar{U}_{0} \times \bar{\Gamma}_{0}\right) \subset N_{1}\left(P_{m}\right) \backslash W_{a}(u)$. Again we shall prove that there is a constant $\gamma \geq 0$, independent of $\left(\bar{x}_{0}, \bar{\xi}_{0}\right) \in\left(U_{0} \times \Gamma_{0}\right) \cap b \cap\{(x, \xi)$; $|\xi|=1\}$, such that if $\left(\bar{x}_{0}, \bar{\xi}_{0}\right) \notin \mathbb{W} F_{a}(u)$ then $b \cap B_{\gamma}\left(\bar{x}_{0}, \bar{\xi}_{0}\right) \cap \mathbb{W} F_{a}(u)=\not$. By making a linear change of coordinates and dividing by a complex constant we can always reduce to the situation that $\bar{x}_{0}=0$ and $d_{\xi} P_{m}\left(\bar{\xi}_{0}\right)=(0, \cdots, 0,1)$, i.e., $b$ is the $x_{n}$-axis. Since $\left(0, \bar{\xi}_{0}\right) \notin \mathrm{WF}_{a}(u)$, there is a conic neighborhood $U \times \Gamma$ of $\left(0, \bar{\xi}_{0}\right)$ such that $(U \times \Gamma) \cap W_{F_{a}}(u)=\varnothing$. We suppose that $U$ is of the form $\left\{x ;\left|x_{n}\right| \leq \epsilon\right.$ and $\left.\left|x^{\prime}\right|<\epsilon\right\}$. Let $\psi_{N}^{\prime}(\xi)$ be homogeneous functions of degree 0 with support in $\Gamma$ and satisfying (1.1) when $|\xi|=1$. Suppose also that $\psi_{N}^{\prime}(\xi)=1$ in a conic neighborhood of $\bar{\xi}_{0}$ and put $\psi_{N}(\xi)=\psi_{N}^{\prime}(\xi) \chi(\xi / N)$, where the functions $\chi_{N}(\xi)$ satisfy (1.1), vanish when $|\xi| \leq 1$ and are equal to 1 when $|\xi| \geq 2$. Then

$$
\left|D_{x}^{a} \psi_{N}(D) u(x)\right| \leq C^{N} N^{|a|}, \quad \text { when }|\alpha| \leq \delta N \text { and } x \in U \text {. }
$$

We can always assume that $U \times \Gamma \subset U_{0} \times \Gamma_{0}$ so, since $\left[P(D), \psi_{N}(D)\right]=0$, it follows that

$$
\left|D_{x}^{\alpha} P(D) \psi_{N}(D) u(x)\right| \leq C^{N} N^{|a|}, \quad \text { when }|\alpha| \leq \delta N \text { and } x \in U
$$

Assume, for simplicity, that $U=\left\{x ;\left|x_{n}\right|<1\right.$ and $\left.\left|x^{\prime}\right|<1\right\}$. Using (5.20) and (5.21), we shall prove that if $0<a<1 / 2$ then (5.20) is valid in a neighborhood of $(0, \cdots, 0, a)$. To do this we employ the change of coordinates (5.17). In the new coordinates $\psi_{N}(D)$ has a symbol $a_{N}(y, \eta)$ given by (2.2). From (5.20) it 
follows that

$$
\left|D_{y}^{a} a_{N}(y, D) u(y)\right| \leq C^{N}|a|, \quad \text { when }|a| \leq \delta N \text { and } y \in G_{1}(\epsilon, M) .
$$

Here $G_{1}(\epsilon, M)=\left\{y ;\left|y^{\prime}\right| \leq \epsilon, 2 M\left|y^{\prime}\right|^{2}-\epsilon<y_{n}<2 M\left|y^{\prime}\right|^{2}+\epsilon\right\}$. We assume that $M$ is so large that $2 a+\epsilon<2 M \epsilon^{2}$ and put $G_{2}(\epsilon, M)=\left\{y ; 2 M\left|y^{\prime}\right|^{2}-\epsilon<y_{n}<2 a\right\}$. Then it follows from (5.22) that

$$
\left|D_{y}^{a} P(y, D) \circ a_{N}(y, D) u(y)\right| \leq C^{N}{ }^{\prime a \mid}, \quad \text { when }|a| \leq \delta N \text { and } y \in G_{2}(\epsilon, M) \text {. }
$$

Since $d_{\xi} P_{m}\left(\xi_{0}\right)=(0, \cdots, 0,1)$ it follows that if the support of $\psi_{N}(\xi)$ is small enough, depending on $M$, then

$$
\left.\partial P_{m}(y, \eta) / \partial \eta_{n} \neq 0, \quad \text { when }(y, \eta) \in\left(\operatorname{supp} a_{N}\right) \cap \overline{\left(G_{2}(\epsilon, M)\right.} \times R^{n}\right) .
$$

We can now apply the factorization lemma of Hörmander to obtain operators $Q_{N}(y, D)$ and $L_{N}(y, D)=\partial / \partial y_{n}+S_{N}\left(y, D^{\prime}\right)$ such that

$$
\left(Q_{N}(y, D) \circ L_{N}(y, D) \circ a_{N}(y, D)-P(y, D) \circ a_{N}(y, D)\right) \in T_{0}\left(G_{2}(\epsilon, M)\right),
$$

and the points in supp $a_{N}$ are noncharacteristic with respect to $\left(Q_{N}\right)$. From $(5.23)$ and (5.25) we get

$$
\left|D_{y}^{a} L_{N}(y, D) \circ a_{N}(y, D) u(y)\right| \leq C^{N}|a|, \quad \text { when }|a| \leq \delta N \text { and } y \in G_{2}(\epsilon, M) \text {. }
$$

It follows from Theorem 1.6.5 of [10] that $a_{N}(y, D) u \in C_{0}^{\left[\delta^{\prime} N\right]}$. Moreover, since $P(D)$ has constant coefficients, $L_{N}$ satisfies the hypothes is of Theorem 4.1. We therefore conclude from Theorem 4.1 , in a finite number of steps, that (5.22) is actually satisfied in $G_{2}(\epsilon, M)$. Finally $b$ is invariant under the coordinate transformation so $(5.20)$ is valid in a neighborhood of $(0, \cdots, 0, a)$. In view of Lemma 3.1 , this completes the proof of Theorem 1.4.

\section{REFERENCES}

1. K. G. Andersson, Propagation of analyticity of solutions of partial differential equations with constant coefficients, Ark. Mat。 8 (1971), 277-302.

2. - Propagation of analyticity for solutions of differential equations of principal type, Bull. Amer. Mtah. Soc. 78 (1972), 479-482.

3. L. Boutet de Monvel and P. Krée, Pseudo-differential operators and Gevrey classes, Ann. Inst. Fourier (Grenoble) 17 (1967), fasc. 1, 295-323. MR $37 \# 1760$.

4. A. P. Calderón, Uniqueness in the Cauchy problem for partial differential equations, Amer. J. Math. 80 (1958), 16-36. MR 21 \#3675.

5. J. J. Duistermaat and L. Hörmander, Fourier integral operators. II, Acta Math. 128 (1972), 183-269.

6. L. Hörmander, Pseudo-differential operators and hypoelliptic equations, Proc. Sympos. Pure Math., vol. 10, Amer. Math. Soc., Providence, R. I., 1967, pp. 138-183.

7. - Fourier integral operators. I, Acta Math. 127 (1971), 79-183. 
8. L. Hörmander, Linear differential operators, Actes Congrès Internat. Math. (Nice, 1970), vol. 1, Gauthier-Villars, Paris, 1971, pp. 121-133.

9. - Uniqueness theorems and wave front sets for solutions of linear differential equations with analytic coefficients, Comm. Pure Appl. Math. 24 (1971), 671-703.

10. - On the existence and regularity of solutions of linear pseudo-differential equations, Enseignement Math. 17 (1971), 99-163.

11. T. Kawai, Construction of local elementary solutions for linear partial differential operators with real analytic coefficients. I. The case with real principal symbols, Publ. Res. Inst. Math. Sci. Kyoto 7 (1971), 363-397.

12. B. Malgrange, Unicité du problène de Cauchy, Séminaire Bourbaki 1lième année: 1958/59, Exposé 178, fasc. 2, Secrétariat mathématique, Paris, 1959. MR 28 \#1091.

13. L. Nirenberg, Pseudo-differential operators, Proc. Sympos. Pure Math., vol. 16, Amer. Math. Soc., Providence, R. I., 1970, pp. 149-167. MR 42 \#108.

14. M. Sato, Hyperfunctions and partial differential equations, Proc. Internat. Conference on Functional Analysis and Related Topics (Tokyo, 1969), Univ. of Tokyo Press, Tokyo, 1970, pp. 91-94.

15. - Regularity of hyperfunction solutions of partial differential equations, Actes Congrès Internat. Math. (Nice, 1970), vol. 2, Gauthier-Villars, Paris, 1971, pp. 785-794.

16. - On pseudo-differential equations in hyper-function theory, Amer. Math. Soc. Sympos. on Partial Differential Equations, Berkeley, 1971.

17. J. J. Duistermaat, On Carleman estimates for pseudo-differential operators, Invent. Math. 17 (1972), 31-43.

DEPARTMENT OF MATHEMATICS, UNIVERSITY OF LUND, LUND, SWEDEN 